Journal for ImmunoTherapy of Cancer

\section{Targeting PARP-1 with metronomic therapy modulates MDSC suppressive function and enhances anti-PD-1 immunotherapy in colon cancer}

To cite: Ghonim MA, Ibba SV, Tarhuni AF, et al. Targeting PARP-1 with metronomic therapy modulates MDSC suppressive function and enhances anti-PD-1 immunotherapy in colon cancer. Journal for ImmunoTherapy of Cancer 2021;9:e001643. doi:10.1136/jitc-2020-001643

- Additional material is published online only. To view please visit the journal online (http://dx.doi.org/10.1136/jitc2020-001643).

MAG, SVI, AFT and YE contributed equally.

Accepted 20 December 2020

Check for updates

(C) Author(s) (or their employer(s)) 2021. Re-use permitted under CC BY-NC. No commercial re-use. See rights and permissions. Published by BMJ.

For numbered affiliations see end of article.

Correspondence to Professor A Hamid Boulares; hboulr@|suhsc.edu

\section{ABSTRACT}

Background Poly(ADP-ribose) polymerase (PARP) inhibitors (eg, olaparib) are effective against BRCAmutated cancers at/near maximum tolerated doses by trapping PARP-1 on damaged chromatin, benefitting only small patient proportions. The benefits of targeting non-DNA repair aspects of PARP with metronomic doses remain unexplored.

Methods Colon epithelial cells or mouse or human bone marrow (BM)-derived-myeloid-derived suppressor cells (MDSCs) were stimulated to assess the effect of partial PARP-1 inhibition on inflammatory gene expression or immune suppression. Mice treated with azoxymethane/ four dextran-sulfate-sodium cycles or $A P C^{\mathrm{Min} /+}$ mice bred into PARP $-1^{+-}$or treated with olaparib were used to examine the role of PARP-1 in colitis-induced or spontaneous colon cancer, respectively. Syngeneic MC38 cell-based (microsatellite instability, MSl ${ }^{\text {high }}$ ) or CT-26 cell-based (microsatellite stable, MSS) tumor models were used to assess the effects of PARP inhibition on host responses and synergy with anti-Programmed cell Death protein (PD)-1 immunotherapy.

Results Partial PARP-1 inhibition, via gene heterozygosity or a moderate dose of olaparib, protected against colitismediated $/ A P C^{\text {Min }}$-mediated intestinal tumorigenesis and $A P C^{\mathrm{Min}}$-associated cachexia, while extensive inhibition, via gene knockout or a high dose of olaparib, was ineffective or aggravating. A sub-IC50-olaparib dose or PARP-1 heterozygosity was sufficient to block tumorigenesis in a syngeneic colon cancer model by modulating the suppressive function, but not intratumoral migration or differentiation, of MDSCs, with concomitant increases in intratumoral T cell function and cytotoxicity, as assessed by granzyme-B/interferon- $\gamma$ levels. Adoptive transfer of WT-BM-MDSCs abolished the protective effects of PARP1 heterozygosity. The mechanism of MDSC modulation involved a reduction in arginase-1/inducible nitric oxide synthase/cyclo-oxygenase-2, but independent of PARP-1 trapping on chromatin. Although a high-concentration olaparib or the high-trapping PARP inhibitor, talazoparib, activated stimulator of interferon gene (STING) in BRCAproficient cells and induced DNA damage, sub-IC50 concentrations of either drug failed to induce activation of the dsDNA break sensor. STING expression appeared dispensable for MDSC suppressive function and was not strictly required for olaparib-mediated effects. Ironically, STING activation blocked human and mouse MDSC function with no additive effects with olaparib. A metronomic dose of olaparib was highly synergistic with anti-PD-1-based immunotherapy, leading to eradication of $\mathrm{MSI}^{\text {high }}$ or reduction of MSS tumors in mice.

Conclusions These results support a paradigm-shifting concept that expands the utility of PARP inhibitor and encourage testing metronomic dosing of PARP inhibitor to enhance the efficacy of checkpoint inhibitor-based immunotherapies in cancer.

\section{BACKGROUND}

Poly(ADP-ribose) polymerase inhibitors (PARPi) (eg, olaparib and talazoparib) are primarily effective against BRCA-defective ovarian or breast cancers, benefitting only a small proportion of patients with cancer. ${ }^{1}$ The ultimate goal of the therapy is maximal inhibition of PARP to achieve synthetic lethality, taking advantage of the inability of BRCA-deficient cancer cells to repair DNA. ${ }^{2}$ A number of clinical trials explored the efficacy of PARPi in the treatment of other cancers with no obvious mutations in BRCA 1. ${ }^{3}$ The role of PARP-1 in DNA repair does not require full activity of the enzyme, as PARP-1 gene heterozygosity, which leads to $\sim 50 \%$ reduction in the levels of the enzyme, or treatment with low concentrations of PARPi is not associated with major defects in DNA repair. ${ }^{4-6}$ The function of PARP-1 in cancer is intimately related to its ability to provide an alternative pathway for cancer cells to survive especially those associated with defects in DNA repair. ${ }^{7}$ The mechanism by which PARP-1 contributes to immune responses may be entirely different. We reported that PARP-1 heterozygosity or a metronomic dose of PARPi substantially blocked atherogenesis in mice ${ }^{8}$ and blocks Th2 
inflammation in allergen-exposed mice. ${ }^{910}$ Altogether, these reports suggest that low-to-moderate doses of PARPi have completely different immunomodulatory effects than those mediated by high doses of the drugs.

Myeloid-derived suppressor cell (MDSC) recruitment and expansion represent critical events during cancer progression by providing an advantage to cancer cells, allowing them to evade the immune system. ${ }^{11}$ MDSCs are specialized in blocking $\mathrm{T}$ cell function by depriving $\mathrm{T}$ cells of essential amino acids through expression of arginase (ARG)-1, producing oxidizing molecules through the expression of inducible nitric oxide synthase (iNOS) or cyclo-oxygenase (COX)-2, as well as blocking $\mathrm{T}$ cell recruitment to tumors. ${ }^{11}$ Therefore, it is clear that interfering with the function and/or recruitment of MDSCs may provide important benefits to strategies targeting cancer cells, representing an attractive strategy to treat not only colon cancer but also many other cancers.

The stimulator of interferon genes (STING) were reported to play an important role in PARPi-mediated tumor cell toxicity in a BRCA deficiency-dependent manner. ${ }^{12}{ }^{13}$ However, the PARPi-STING relationship was also reported in BRCA-proficient cells. ${ }^{14}$ The common link in these studies is the requirement for DNA damage, which can only be induced by high concentrations of PARPi. The STING pathway was also shown to be involved in MDSC differentiation and antitumor immune responses. ${ }^{1516}$ A primary goal of the current study was to explore the effects of partial PARP inhibition achieved by gene heterozygosity or a metronomic dose of olaparib on different models of colon cancer and to examine whether such approaches exert different immune responses to carcinogenesis by focusing primarily on MDSCs. We also examine whether the effects of PARP inhibition on MDSC function would synergize with anti-Programmed cell Death protein (PD)-1 immunotherapy in microsatellite instability ( $\mathrm{MSI}^{\text {high }}$ ) and microsatellite stable (MSS) colon cancer mouse models.

\section{MATERIALS AND METHODS}

Details of the materials and methods used in this study are outlined in online supplemental materials and methods.

\section{Animals and genotyping}

Mice were housed in a specific pathogen-free facility at Louisiana State University Health Sciences Center. The different mouse strains were genotyped using standard protocols (online supplemental table S1 and figure S6).

\section{Cell isolation, culture, treatments, RNA extraction, complementary DNA synthesis and quantitative PCR}

Primary colon epithelial cells (CECs) ${ }^{17}$ and the different cell lines were maintained as described in online supplemental materials and methods. Total RNA was analyzed by Real-Time (RT)-quantitative PCR with previously validated primer sets (online supplemental table S1). ${ }^{18-20}$ MDSCs were generated by incubating bone marrow
(BM)-derived hematopoietic stem cells with granulocytemacrophage colony-stimulating factor (GM-CSF), Granulocyte colony-stimulating factor (G-CSF), and interleukin 6 (IL-6). Tumor infiltrating MDSCs were isolated by enzymatic digestion of engrafted tumors followed by selection of the $\mathrm{CD} 11^{+} \mathrm{Gr}-1^{+}$subset. For some experiments, $\mathrm{CD} 45^{+}$ cells were isolated as mixed immune cell populations. For the suppression assay, tumor-derived or BM-derived MDSCs were cocultured with CD3/CD28-stimulated carboxyfluorescein succinimidyl ester (CFSE)-labeled T cells, and proliferation was assessed by flow cytometry.

\section{Tumor models}

The colitis-induced (azoxymethane (AOM)/dextran sulfate sodium (DSS)), $A P C^{\mathrm{Min} /+}$, and syngeneic tumor models are described in detail in online supplemental materials and methods.

\section{Tissue processing, immunohistochemistry, immunofluorescence, cytokines measurement, and FACS analysis}

Serial tissue sections were subjected to H\&E staining, immunohistochemistry or immunofluorescence, as described, ${ }^{21}$ with relevant antibodies. ${ }^{22}$ Sera of mice were assessed for tumor necrosis factor- $\alpha$ (TNF- $\alpha$ ), IL-6, and monocyte chemoattractant protein MCP)-1 by ELISA. Single-cell suspensions obtained from digested tumors were subjected to fluorescence-activated cell sorting (FACS) analysis using the fluorescently labeled antibodies. The details of the gating strategy are described in online supplemental figure S7.

\section{Cell fractionation, protein extraction and immunoblot analysis}

Tumor tissues and single cells were homogenized using standard protocols. MDSCs and Jurkat cells were subjected after treatments to nuclear fractionation as described, ${ }^{23}$ and the remaining chromatin pellets were sonicated in lysis buffer. Protein extracts were subjected to immunoblot analysis, ${ }^{10}$ with the relevant antibodies described in figure legends (also see online supplemental materials and methods).

\section{Statistical analysis}

All data are presented as mean \pm SEM. Analysis of variance for the different groups (same experiment) was conducted using a one-way analysis of variance followed by Tukey's multiple comparison test. When a comparison is conducted between two groups, an unpaired Student's t-test was used. These analyses were facilitated by the PRISM software (GraphPad, San Diego, California).

\section{RESULTS}

Partial PARP-1 inhibition achieves better protection against colitis-driven colon tumorigenesis than complete or extensive inhibition

PARP-1 gene heterozygosity in primary CECs reduced PARP-1 expression by $~ 50 \%$ compared with WT cells (figure 1A). Figure 1B shows that lipopolysaccharide (LPS)-induced expression of IL-6, TNF- $\alpha$, iNOS, 
$\mathbf{A}$
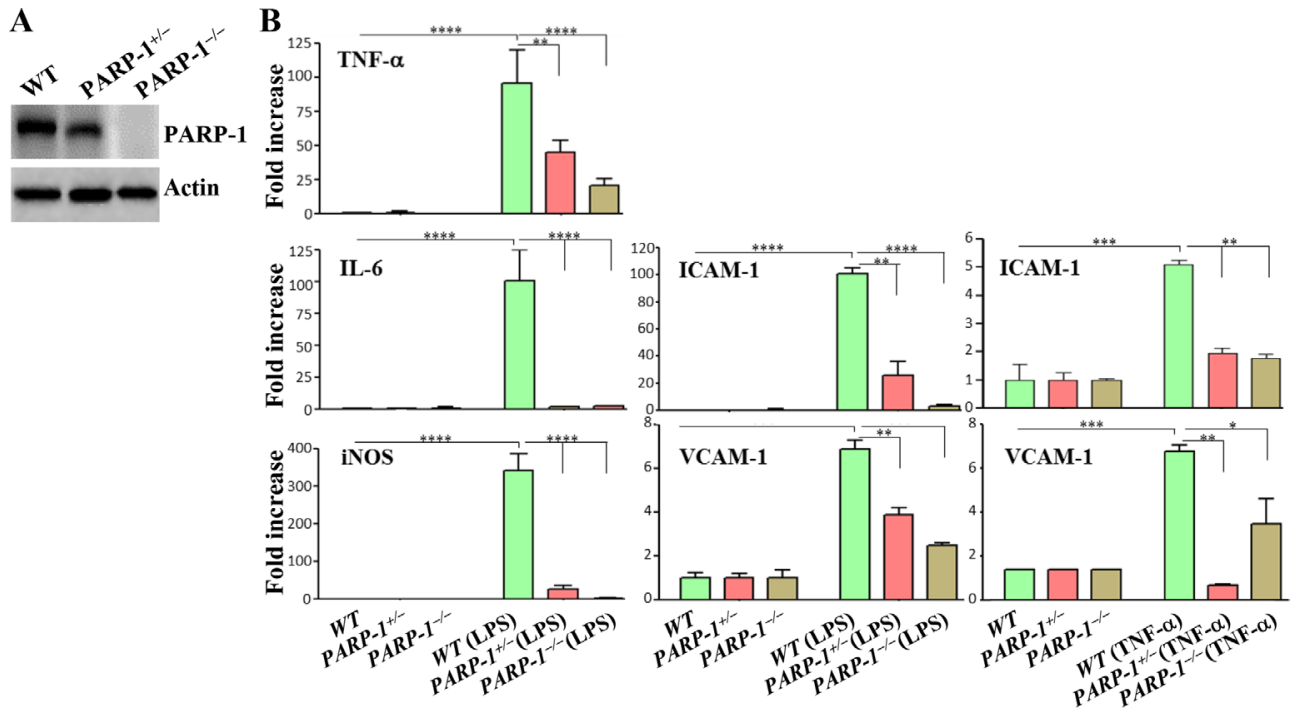

C

D

$\mathbf{E}$
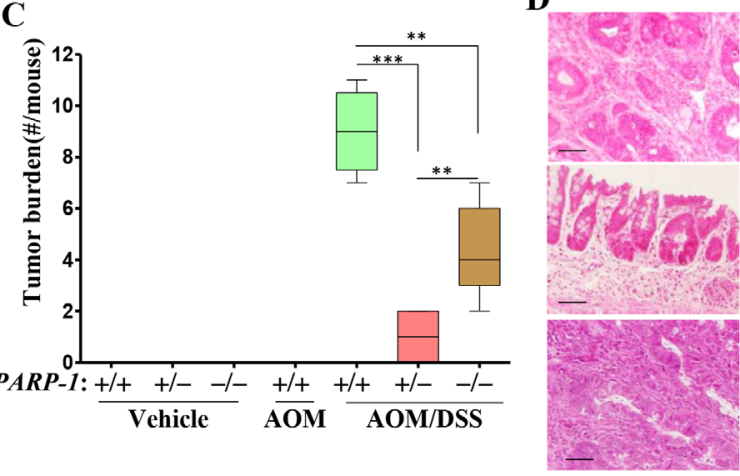

E

F
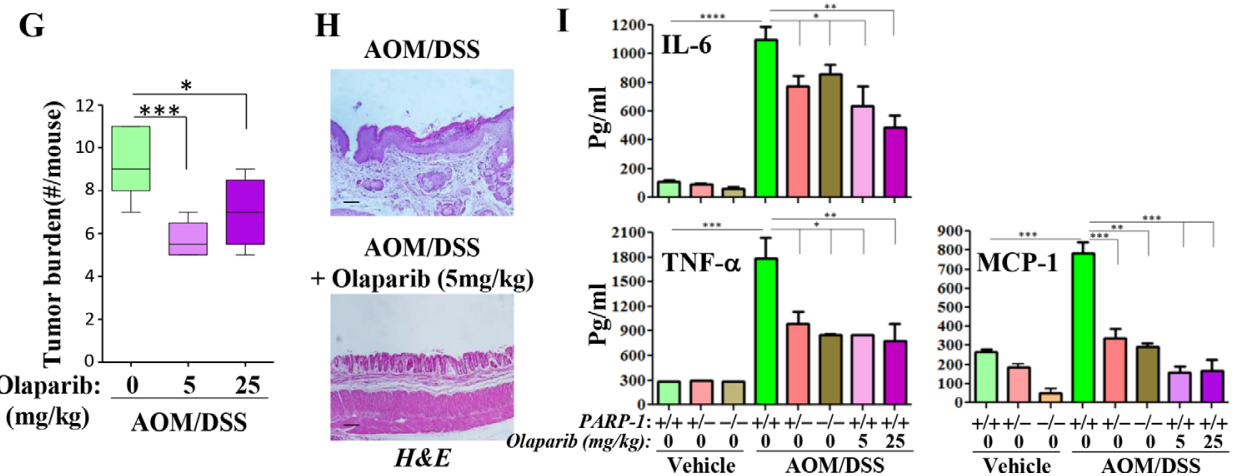

Figure 1 Partial PARP-1 inhibition is sufficient to block chronic inflammation and associated colon tumorigenesis. (A) CECs were isolated from WT, $P A R P-1^{+/-}$or $P A R P-1^{-/-}$mice. Total protein extracts were subjected to immunoblot analysis with antibodies to PARP-1 or actin. (B) CECs were treated with $2 \mu \mathrm{g} / \mathrm{mL}$ LPS or $10 \mathrm{ng} / \mathrm{mL}$ TNF- $\alpha$ for 6 hours, after which RNA was extracted; complementary DNAs were subjected to real-time PCR using sets of primers for mouse TNF- $\alpha$, IL-6, ICAM1 or $\beta$-actin. Fold changes ( $\triangle \triangle \mathrm{CT}$ values) were then calculated using $\beta$-actin as a normalization control. These experiments were conducted in triplicates. (C) WT, $P A R P-1^{+/-}$and $P A R P-1^{-/-}$mice $(\mathrm{n}>6)$ received $10 \mathrm{mg} / \mathrm{kg}$ of AOM intraperitoneally once, followed by four cycles of $1.25 \%$ DSS in drinking water. At 21 weeks of age, mice were sacrificed and colon tumor burden was assessed. (D) H\&E staining of colon tumor sections from the different experimental groups. (E) IHC with antibodies to PCNA. (F) H\&E staining showing the protective effect of PARP-1 gene heterozygosity and knockout against AOM/DSS-induced colitis. (G) WT mice were subjected to AOM/DSS protocol as described above. Mice from all experimental groups ( $\mathrm{n}>6$ ) received intraperitoneal injections of 5 or $25 \mathrm{mg} / \mathrm{kg}$ olaparib or a vehicle twice a week immediately after AOM administration and until a day prior to sacrifice (21 weeks). Colon tumor numbers were counted. $(H)$ H\&E staining showing the protective effect of PARP-1 inhibition by olaparib against AOM/DSS-induced colitis. (I) Sera from the different experimental groups were assessed for IL-6, TNF- $\alpha$ or MCP-1 using sandwich ELISA. For B, C, G, and I: ${ }^{*} \mathrm{p} \leq 0.05,{ }^{* *} \mathrm{p} \leq 0.01,{ }^{* * *} \mathrm{p} \leq 0.001,{ }^{* * *} \mathrm{p} \leq 0.0001$. Bar $=50 \mu \mathrm{m} . \Delta \Delta \mathrm{CT}, \Delta \Delta$ cycle threshold;AOM, azoxymethane; CECs, colon epithelial cells; DSS, dextran sulfate sodium; IHC, immunohistochemistry; IL-6, interleukin 6; ICAM-1, ntercellular adhesion molecule-1; iNOS, inducible nitric oxide synthase; MCP-1, monocyte chemoattractant protein; PARP, poly(ADP-ribose) polymerase; PCNA, proliferating cell nuclear antigen; TNF- $\alpha$, tumor necrosis factor- $\alpha$; VCAM-1, vascular cell adhesion molecule; WT, wild type. 
intercellular adhesion molecule (ICAM)-1, and vascular cell adhesion molecule (VCAM)-1 in WT CECs was efficiently reduced by PARP-1 heterozygosity or knockout. Remarkably, PARP-1 heterozygosity was as effective as knockout in reducing or blocking the expression of the examined genes. In response to TNF- $\alpha$, PARP-1 heterozygosity was either equally effective or even better than knockout in reducing the expression of ICAM-1 and VCAM-1, respectively (figure 1B). When considering that synthetic lethality in BRCA-deficient cells requires maximum PARP inhibition, the effects of partial inhibition on inflammatory or immune factors entail a completely different and DNA repair-independent mechanism(s).

To begin testing the above-mentioned concept, we used the inflammation-driven AOM/DSS-based colon cancer model. AOM/DSS-treated PARP-1 $1^{-/-}$mice displayed a significantly lower tumor burden than similarly treated WT mice (figure 1C), consistent with a recent report. ${ }^{24}$ However, partial PARP-1 inhibition generated by gene heterozygosity was more effective. The tumors detected in all groups were small $(\sim 2 \mathrm{~mm}$ in diameter $)$, reaching adenoma or adenocarcinoma status $(>1 \mathrm{~mm})$ only in AOM/DSS-treated WT and PARP-1 $1^{-/}$mice. The colon of AOM/DSS-treated WT mice displayed typical colon tumors with marked hyperplasia and dysplasia and development of large aberrant crypt foci (ACF), which were not drastically different from those of similarly treated $P A R P-1^{-/-}$mice (figure 1D). Conversely, the tumors from $\mathrm{AOM} / \mathrm{DSS}$-treated $P A R P-1^{+/-}$mice were smaller and consisted mainly of large ACFs. High immunoreactivity to proliferating cell nuclear antigen (PCNA), a marker of cell proliferation, was detected in tumors of AOM/DSStreated WT and $P A R P-1^{-/}$mice (figure $1 \mathrm{E}$ ), and this was much lower in tumors of treated $P A R P-I^{+/-}$mice (online supplemental figure S1). AOM/DSS treatment-induced damage to the colonic mucosa in $P A R P-1^{+/-}$and PARP$1^{-/-}$mice was not as prevalent as that observed in similarly treated WT mice (figure $1 \mathrm{~F}$ ). It is noteworthy that the mucosa of AOM/DSS-treated PARP-1 ${ }^{+/-}$or PARP-1 $1^{-/}$ mice showed some disorganization and injury, but the colonic crypts were relatively intact or appear to be in the process of recovery. These results are consistent with a previous report. ${ }^{25}$

For pharmacological studies, we elected to be consistent with our previous reports and use the moderate olaparib dose of $5 \mathrm{mg} / \mathrm{kg}$ in addition to a dose that is five times higher $(25 \mathrm{mg} / \mathrm{kg})$. Treatment with olaparib at either dose significantly reduced tumor burden and accompanying colitis compared with treatment with the vehicle (figure 1G,H). Figure 1I shows that all forms of PARP-1 inhibition were associated with a marked reduction in the levels of TNF- $\alpha$ and MCP-1 but a moderate effect on IL-6 levels. These results suggest that the role of PARP-1 in colon inflammation may constitute, in part, the underlying mechanism by which the enzyme participates in the pathogenesis of colon cancer.
Partial PARP-1 inhibition protects against, while complete inhibition aggravates, $A P C^{\mathrm{Min}}$-induced tumor burden in mice We next examined the role of PARP-1 in colon tumorigenesis in the spontaneous $A P C^{M i n}$-based mouse model. PARP-1 heterozygosity provided remarkable protection against tumor development (figure 2A). Surprisingly, PARP-1 knockout not only failed to provide any protection but actually significantly aggravated tumor burden. In addition, PARP-1 heterozygosity completely blocked the generation of large tumors $(>4 \mathrm{~mm})$ with a significant concomitant decrease in small-sized $(<2 \mathrm{~mm})$ and medium-sized (2-4 mm) tumors (figure 2B). Conversely, PARP-1 knockout promoted an increase in the number of small tumors. PCNA immunoreactivity in tumors of $A P C^{M i n /+}$ mice did not differ from that observed in $A P C^{\mathrm{Min} /} \mathrm{PARP}-\mathrm{1}^{-/-}$mice (figure $2 \mathrm{C}$ ), but the difference between these two groups and that of $P A R P-1^{+/-}$mice was more pronounced. Paradoxically, intratumoral inflammation as assessed by immunoreactivity to COX-2 (figure 2D) was equally reduced in tumors of $P A R P-1^{+/}$and $P A R P$ $1^{-/-}$mice. PARP inhibition from the moderate dose of olaparib $(5 \mathrm{mg} / \mathrm{kg})$ provided good protection against tumor development. Although the higher dose resulted in great variability in response, overall it provided no significant protection against tumor burden (figure 2E). While PARP-1 heterozygosity completely prevented $A P C^{M i n}$-induced cachexia, PARP-1 knockout prevented such weight loss only to a small level, although it aggravated tumor burden in $A P C^{\mathrm{Min} /+}$ mice (figure $2 \mathrm{~F}$ ). While the moderate dose of olaparib protected against cachexia in $A P C^{M i n /+}$ mice, the high dose did not. As expected, $A P C^{\mathrm{Min} / \mathrm{+}}$ mice displayed splenomegaly (figure $2 \mathrm{G}$ ) and systemic inflammation (figure $2 \mathrm{H}$ ), as assessed by the increased levels of MCP-1, TNF- $\alpha$, and IL- 6 . All forms of PARP-1 inhibition reduced the size of spleens and MCP-1 and TNF- $\alpha$ levels. IL- 6 levels remained high. These results suggest that the protective effects of partial PARP-1 inhibition are not strictly associated with a modulation in systemic inflammation, but may also be related to host responses, including that of the immune system, to inflammation and tumorigenesis.

\section{A metronomic dose of olaparib or PARP-1 gene heterozygosity is sufficient to promote a tumor-suppressive environment in mice}

We took advantage of a widely used syngeneic model based on the colon adenocarcinoma cell line MC-38, which represents $\mathrm{MSI}^{\text {high }}$ colon cancer. ${ }^{26}$ It is noteworthy that MC-38 cells express high levels of PARP-1 (figure 3A). Engraftment of MC-38 cells into the flanks of WT mice led to sizeable tumors (figure $3 \mathrm{~B}$ ), while engraftment into either $P A R P-1^{+/-}$or $P A R P-1^{-/-}$mice led to significantly smaller tumors displaying little inflammation as assessed by ICAM-1-immunoreactivity (figure 3C). These effects were accompanied by a maintenance of normal-sized spleens (figure 3D). Because a moderate dose of $5 \mathrm{mg} /$ $\mathrm{kg}$ olaparib provided superior protection compared with a high dose in the $A P C^{M i n /+}$ mouse model, we continued 
A

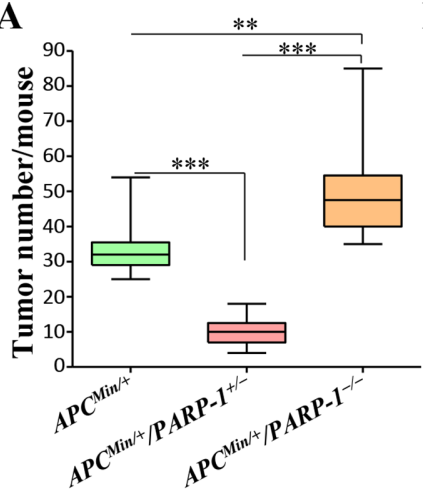

B

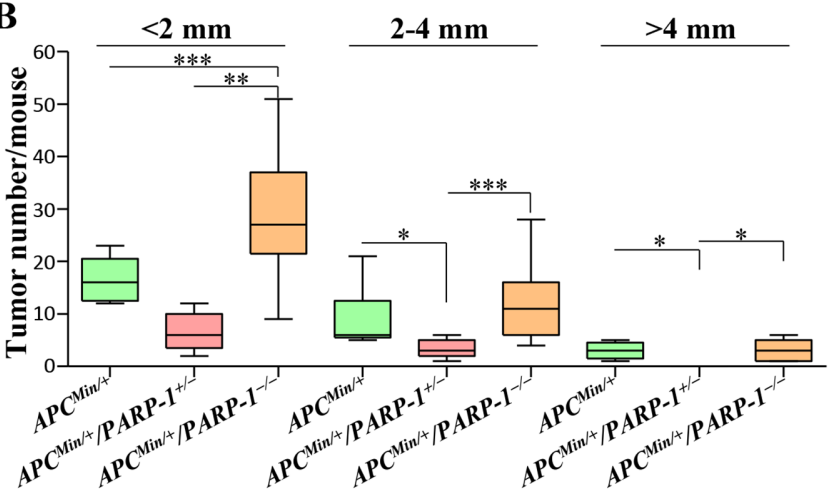

C IHC: PCNA

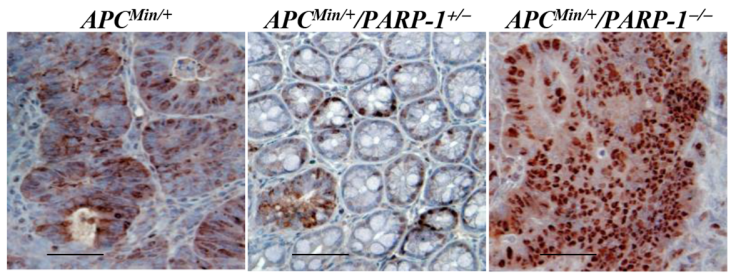

D IHC: COX2

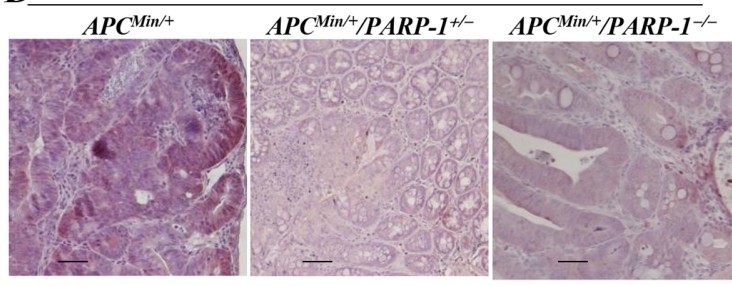

$\mathbf{E}$

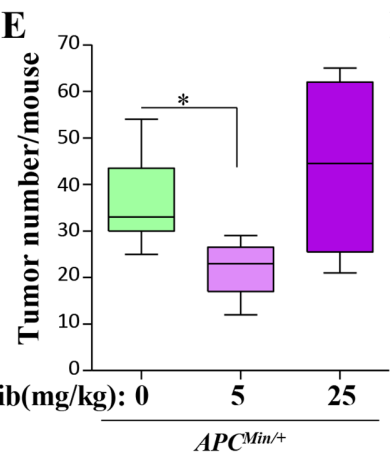

F

G

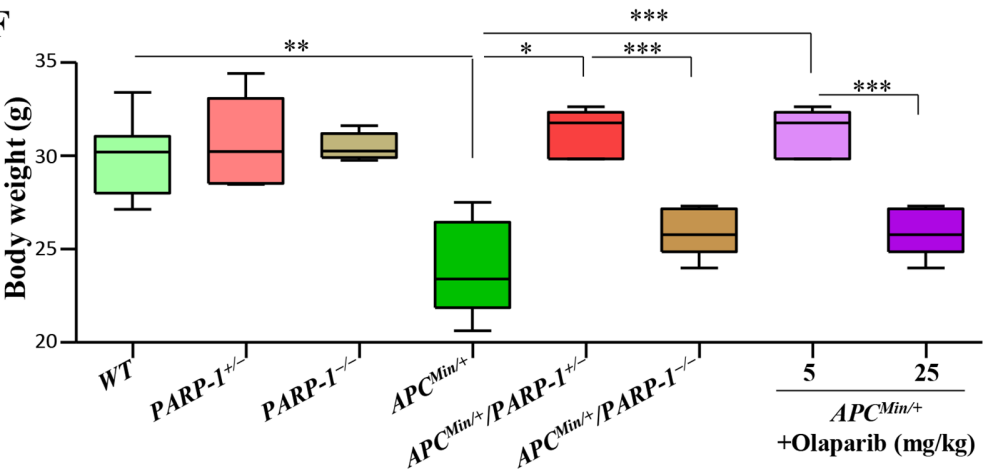

$\operatorname{Olaparib(\mathrm {mg}/\mathrm {kg}):\frac {1}{0}\quad \frac {1}{APC^{\text {Min}}+}} 2$
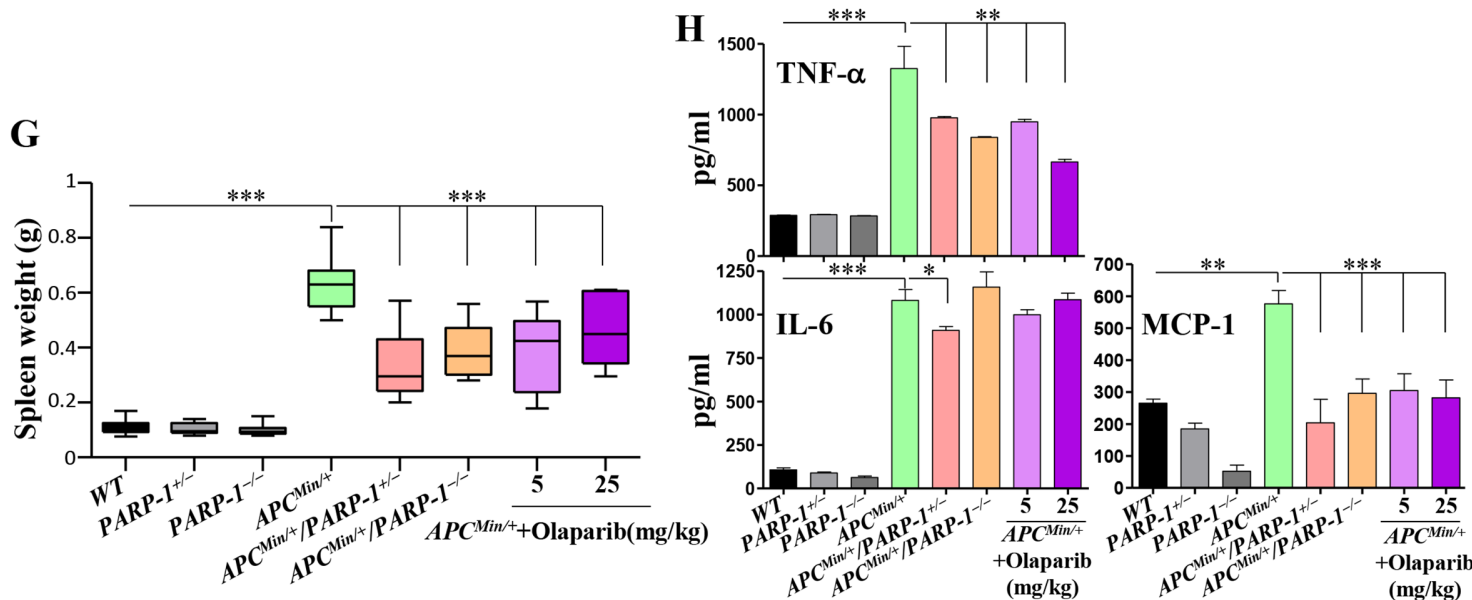

Figure 2 Partial inhibition of PARP-1 protects, while complete inhibition is ineffective, against $A P C^{\text {Min }}$-induced tumor burden in mice. (A) $A P C^{\mathrm{Min} /+}, A P C^{\mathrm{Min} /+} P A R P-1^{+/-}$, and $A P C^{\mathrm{Min} /+} P A R P-1^{-/-}$mice $(\mathrm{n}>10)$ were sacrificed at 16 weeks of age. Tumor numbers were then counted. (B) Tumor burden was analyzed based on size and divided into groups with tumors lower than $2 \mathrm{~mm}$, 2-4 $\mathrm{mm}$, and bigger than $4 \mathrm{~mm}$. Colon tumor sections from the three different groups were subjected to IHC with antibodies specific to PCNA (C) or COX-2 (D). (E) $A P C^{\text {Min/+ }}$ mice were randomized into three groups $(n>6)$ and received, intraperitoneally, 5 or $25 \mathrm{mg} / \mathrm{kg}$ olaparib twice a week, or vehicle $(0.005 \%$ DMSO in saline) from 5 weeks up to 16 weeks of age. Mice were then sacrificed and tumor burden was quantified. Total body (F) or spleen (G) weight of mice from the different groups at 16 weeks of age. (H) Sera from the different mouse groups were assessed for TNF- $\alpha$, IL-6, or MCP-1 using sandwich ELISA. For A, B, E-H: ${ }^{*} \mathrm{p} \leq 0.05,{ }^{* \star} \mathrm{p} \leq 0.01,{ }^{* \star *} \mathrm{p} \leq 0.001$. Bar $=50 \mu \mathrm{m}$. COX-2, cyclo-oxygenase-2; DMSO, dimethyl sulfoxide ; IHC, immunohistochemistry; IL-6, interleukin 6; MCP-1, monocyte chemoattractant protein; PARP, poly(ADP-ribose) polymerase; PCNA, proliferating cell nuclear antigen; TNF- $\alpha$, tumor necrosis factor- $\alpha$; WT, wild type. 
A

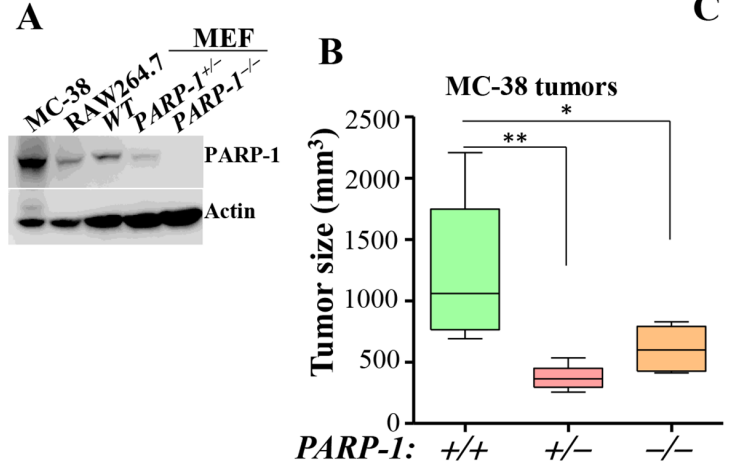

D

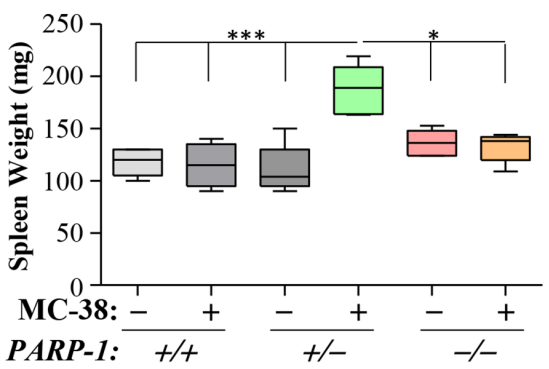

F

Olaparib (mg/kg)

IHC: ICAM-1

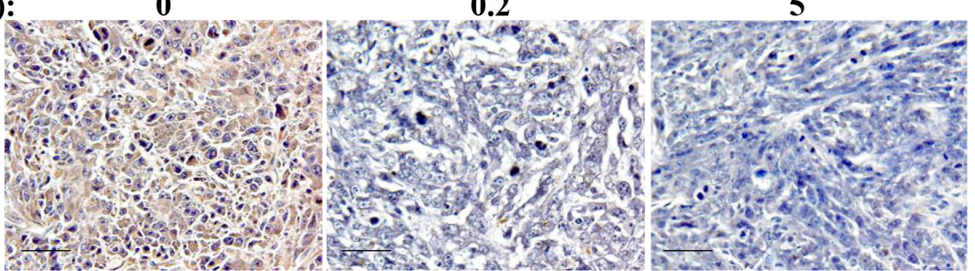

C

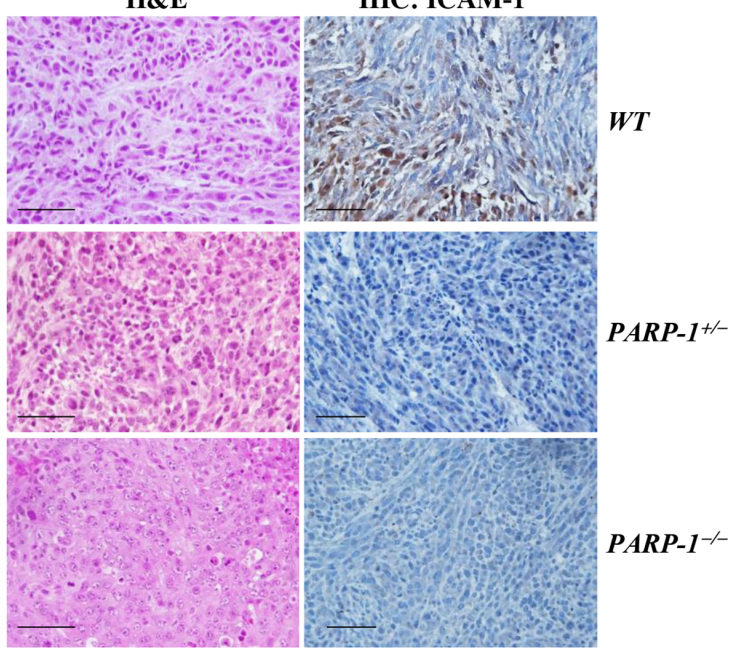

E

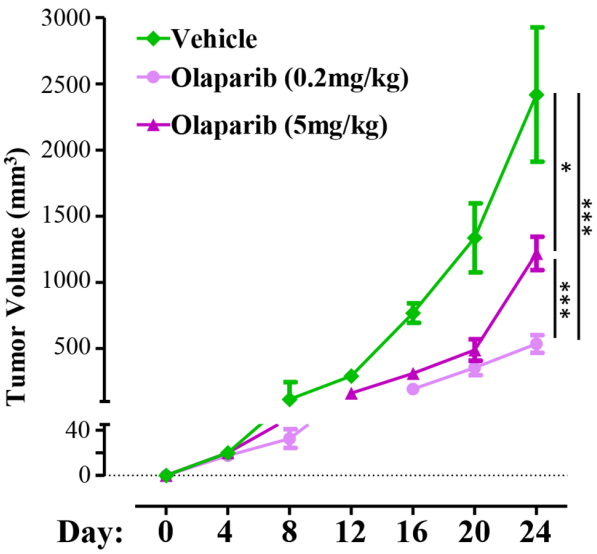

Figure 3 A metronomic dose of olaparib or PARP-1 heterozygosity is sufficient to promote a tumor-suppressive environment in a syngeneic colon cancer mouse model. (A) Protein extracts from MC-38 cells, RAW264.7 cells, or mouse embryonic fibroblasts derived from WT, PARP- $1^{+/-}$, or PARP-1 $1^{-/-}$mice were subjected to immunoblot analysis. (B) MC-38 cells $\left(2.5 \times 10^{5}\right)$ were injected subcutaneously into the left flanks of WT, PARP-1 $1^{+/-}$or PARP $-1^{-/-}$mice $(n>6)$. Tumor sizes were measured on day 21. (C) Mice were sacrificed and tumor sections were subjected to H\&E (left panels) or IHC staining (right panels) with antibodies to ICAM-1. (D) Spleen weight of mice from the different groups. (E) MC-38 cells were engrafted into WT mice ( $\mathrm{n}>6$ per group). When tumors became palpable $\left(\sim 20 \mathrm{~mm}^{3}\right)$, mice received different doses of olaparib or vehicle. Tumor volume was measured on the indicated days. (F) Mice were sacrificed on day 24 and tumor sections were subjected to IHC staining with antibodies to ICAM-1. (G) Sera from the different mouse groups were assessed for TNF- $\alpha$, IL- 6 or MCP- 1 by ELISA. For B, D-E, and G: ${ }^{*} p \leq 0.05,{ }^{* *} p \leq 0.01$, ${ }^{\star \star \star} \mathrm{p} \leq 0.001$. Bar=50 $\mu \mathrm{m}$. ICAM-1, ntercellular adhesion molecule-1; IHC, immunohistochemistry; IL-6, interleukin 6; MCP-1, monocyte chemoattractant protein; MEF, mouse embryonic fibroblasts; PARP, poly(ADP-ribose) polymerase; TNF- $\alpha$, tumor necrosis factor- $\alpha$; WT, wild type. 
using it, but also examined the effects of $0.2 \mathrm{mg} / \mathrm{kg}$, a 25-fold lower metronomic dose. Although the moderate dose of olaparib significantly reduced the size of MC-38 cell-based tumors, the lower dose was more effective (figure 3E). Both doses promoted a reduction in intratumoral inflammation (figure 3F). Systemic inflammation was moderately reduced by either olaparib dose, despite the different outcomes on tumor burden (figure 3G). Overall, these results suggest that a low dose of olaparib may exert a superior antitumor effect compared with a high dose despite the relatively similar reduction in intratumoral and systemic inflammation.

\section{PARP-1 plays a key role in MDSC function, and partial inhibition of PARP-1 by gene heterozygosity or low-to- moderate doses of olaparib are sufficient to block the suppressive activity of these cells}

The $0.2 \mathrm{mg} / \mathrm{kg}$ olaparib dose did not significantly affect the frequency of tumor infiltrating MDSCs (figure 4A,B). The $5 \mathrm{mg} / \mathrm{kg}$ olaparib dose, however, significantly increased the numbers and percentages of M-MDSCs (figure 4C). PARP-1 heterozygosity was associated with a minor but statistically significant increase in the percentage of $\mathrm{CD} 11 \mathrm{~b}^{+} \mathrm{ly} 6 \mathrm{C}^{+}$MDSC subset, with a concomitant decrease in the percentage of $\mathrm{CD} 11 \mathrm{~b}^{+} l y 6 \mathrm{G}^{+}$MDSCs. The overall cell numbers, however, were comparable with those detected in tumors on WT mice (figure 4C). Only the low dose of olaparib increased $\mathrm{CD} 3^{+} \mathrm{T}$ cells and most of these were $\mathrm{CD} 8^{+} \mathrm{T}$ cells (figure $4 \mathrm{D}$ ). The spatial distribution of intratumoral MDSCs $\left(\mathrm{Gr}^{+}\right)$and $\mathrm{CD}^{+}$cells in the different groups was corroborated using immunohistochemistry and immunofluorescence (figure 4E). Interestingly, in the spleens, only the moderate dose of olaparib promoted a substantial decrease in overall $\mathrm{CD}^{+} \mathrm{T}$ cells and a concomitant decrease in $\mathrm{CD}^{+} \mathrm{T}$ cells (figure $4 \mathrm{~F}$ ).

Given the possibility that MDSC migration into the tumors may not be sufficiently affected by PARP-1 inhibition to explain the reduction in tumor burden, we speculated that the suppressive function of MDSCs may be influenced. While MDSCs isolated from tumors of WT mice were effective in suppressing the proliferation of CD3/CD28-activated $\mathrm{T}$ cells, MDSCs derived from tumors of olaparib-treated mice exhibited a reduced suppressive capacity (figure 4G). Tumor-derived PARP$1^{+>-}$(figure 4G) and PARP-1 $1^{-/-}$MDSCs were completely non-functional (online supplemental figure S2A). These results suggest that PARP-1 plays a critical role in MDSC function and that these cells are very sensitive to PARP-1 inhibition.

\section{A sub-IC50 concentration of olaparib is sufficient to reduce MDSC suppressive function in vitro, in part by blocking the expression of ARG-1, iNOS, and COX-2, and the adoptive transfer of WT MDSCs abrogates the protective effects of PARP-1 heterozygosity on tumor burden}

BM-derived stem cells were stimulated with GM-CSF, G-CSF, and IL-6. After 24 hours, a portion of WT cells were treated once with increasing concentrations of olaparib and incubated for an additional 3 days. We used $4 \mathrm{nM}$ olaparib as a sub-half maximal inhibitory concentration(IC50) concentration; 0.1 and $5 \mu \mathrm{M}$ olaparib were used as the moderate and high concentrations, respectively. The viability of MDSCs was not affected by any condition (online supplemental figure S2B). While PARP-1 inhibition by a low or moderate olaparib concentration exerted no effect on the percentage of $\mathrm{CD} 11 \mathrm{~b}^{+} \mathrm{Gr} 1^{+}$ MDSCs, the high concentration $(5 \mu \mathrm{M})$ increased such percentage (figure 5A). PARP-1 heterozygosity and knockout caused similar divergent effects (online supplemental figure S2C). The prevalence of MDSCs in tumors of $A P C^{\mathrm{Min} /{ }^{+}} P A R P-1^{-/-}$mice was also higher than that in tumors of $A P C^{M i n /+}$ or $A P C^{M i n /+} P A R P-1^{+/-}$mice (online supplemental figure S2D,E). The suppressive activity of WT-BM-MDSCs was significantly reduced by olaparib even at the sub-IC50 concentration (figure 5B). The moderate and high concentrations blocked the function of MDSCs completely, and this result was mirrored by PARP-1 heterozygosity. The low concentration of olaparib exerted no effect on $\mathrm{T}$ cell proliferation (figure $5 \mathrm{C}$ ); however, the moderate and high concentrations of olaparib reduced such proliferation (online supplemental figure S3). The effect of PARP inhibition on MDSC function was confirmed using ex vivo differentiated cells derived from human BM (figure 5C).

To determine whether the effects of PARP inhibition on MDSCs were, in part, responsible for the effects on tumorigenesis, we adoptively transferred WT-BM-MDSCs into the periphery of MC-38 cell-based tumors in PARP$1^{+/-}$mice; this approach was taken since olaparib is not appropriate to address the question. Figure 5D shows that adoptive transfer of MDSCs into WT mice exerted no effect on tumor size, which is consistent with a recent report. ${ }^{27}$ Administration of WT MDSCs into tumors of $P A R P-1^{+/-}$mice, however, temporally abrogated the effects of PARP-1 heterozygosity, resulting in increased tumor size. The tumors tended to shrink to the level observed in control $P A R P-1^{+/-}$mice a few days later and a second administration of MDSCs reversed the tumor size to that observed in tumors of WT mice. To provide additional support to the above results, we used PARP-1-floxed mice under the control of Tie2/Tek-Cre recombinase, which targets the deletion in hematopoietic cells, and are termed PARP- $1^{\text {Tek-fl/wt }}$ and PARP-1 ${ }^{\text {Tek-fl/fl }}$ for heterozygous and knockout, respectively (online supplemental figure S4A). Online supplemental figure S4B shows that TekCre-driven PARP-1 heterozygosity was sufficient to block MC-38 cell-based tumor formation in mice.

An examination of protein extracts from $\mathrm{CD} 45^{+}$ immune cells isolated from MC-38 cell-based tumors of the different experimental groups by immunoblot analysis revealed that the tumor suppression capacity of these cells, which include MDSCs, was reduced by olaparib treatment at the metronomic low or moderate dose, as evidenced by the reduced expression of ARG-1 (figure 5E,F). Interestingly, the lower levels of ARG-1 were accompanied by a significant increase in the levels of $\mathrm{T}$ 

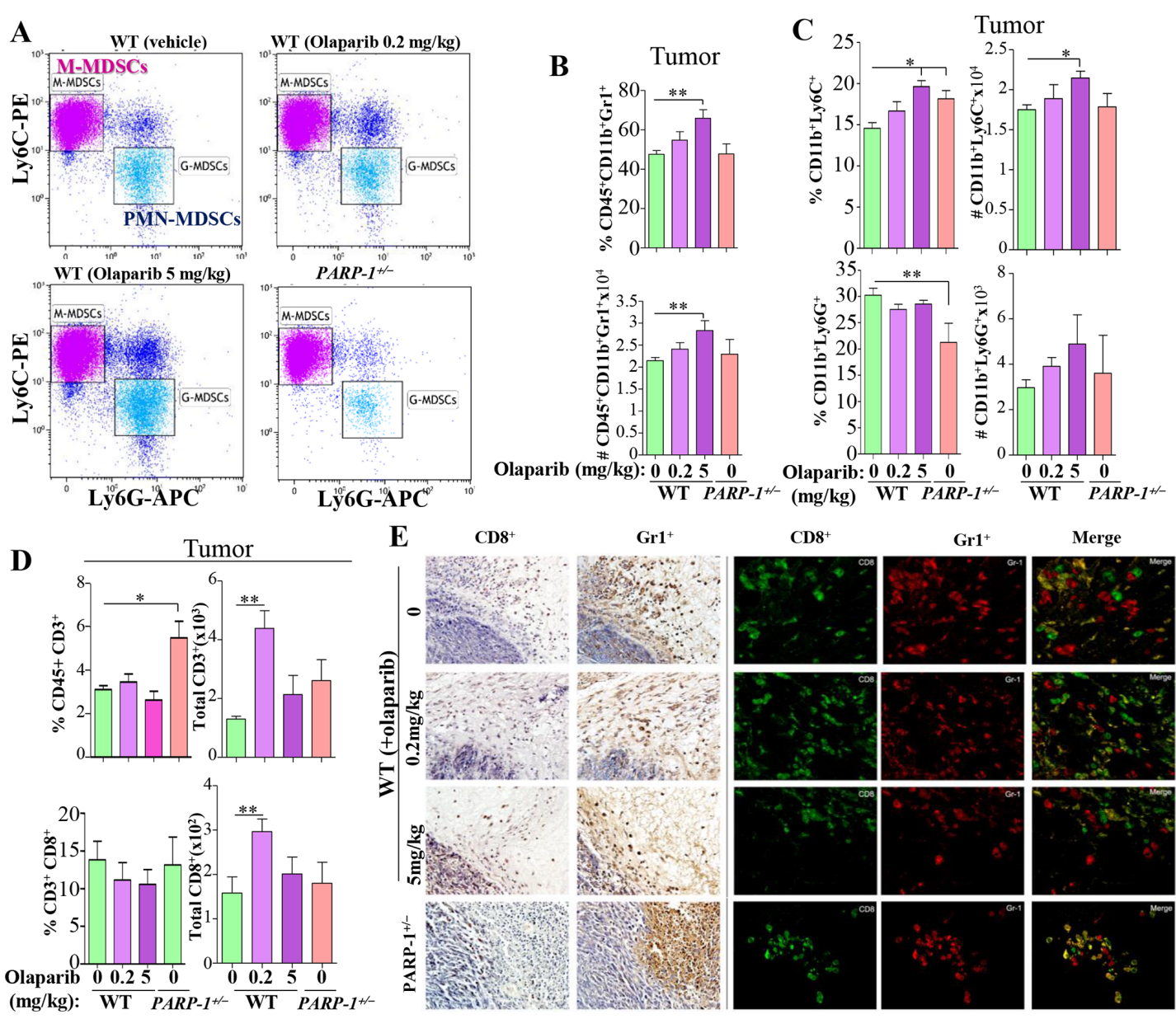

$\mathrm{CD8}^{+}$
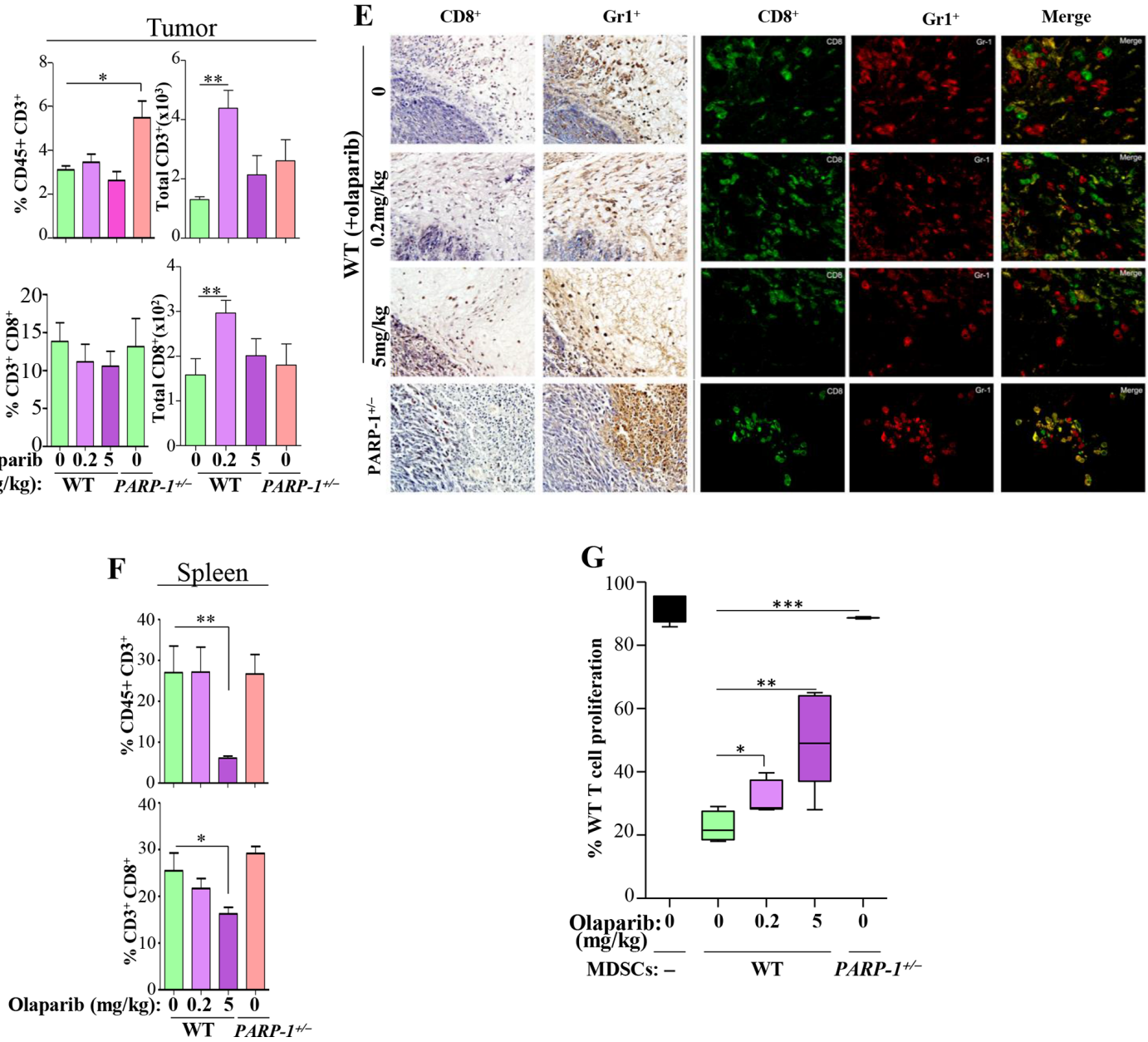

Figure 4 The suppressive function of MDSCs is highly sensitive to PARP inhibition. Tumors from the different experimental groups $(n>6)$ were either processed to generate single-cell suspensions or fixed with formalin. Single-cell suspensions were stained with a combination of antibodies to CD45, CD11b, Gr1, Ly6C, and Ly6G. CD11b+ cell population was gated from the live $\mathrm{CD}_{4} 5^{+}$population. (A) Representative FACS dot plot of the experimental groups. (B) Absolute numbers and percentages of $\mathrm{CD} 11 \mathrm{~b}^{+} \mathrm{Gr} 1^{+}$cell populations in tumors of the different groups. (C) Absolute numbers and percentages of CD11 $\mathrm{b}^{+} \mathrm{Ly} 6 \mathrm{C}^{+}$ and $C D 11 b^{+}$Ly6G $G^{+}$populations in tumors of the different groups. (D) Absolute numbers and percentages of $C D 45^{+} C D 3^{+}$and $\mathrm{CD}^{+} \mathrm{CD}^{+}$cell populations in tumors of the different groups. (E) Serial sections of tumors from WT mice that were treated with 0.2 or $5 \mathrm{mg} / \mathrm{kg}$ olaparib or vehicle or PARP-1+- mice were subjected to IHC (left panels) or immunofluorescence (right panels) with antibodies to mouse Gr1 or CD8. (F) Percentages of $\mathrm{CD} 45^{+} \mathrm{CD} 3^{+}$and $\mathrm{CD} 3^{+} \mathrm{CD} 8^{+}$cell populations in spleens of the different groups. (G) CD11 $\mathrm{b}^{+}$-enriched MDSCs isolated from tumors of WT mice that were treated with $0.2 \mathrm{or} 5 \mathrm{mg} / \mathrm{kg}$ olaparib or vehicle or PARP-1 $1^{+/-}$mice $(n=5)$ were assessed for their ability to suppress proliferation of CD3/CD28-stimulated and CFSClabeled WT CD3 ${ }^{+} \mathrm{T}$ cells at an MDSC to T cell ratio of 1:4. T cell proliferation was assessed by FACS. For B-D, F and G: ${ }^{*} \mathrm{p} \leq 0.05,{ }^{* *} \mathrm{p} \leq 0.01,{ }^{* * *} \mathrm{p} \leq 0.001$. CFSE, carboxyfluorescein succinimidyl ester; FACS, fluorescence-activated cell sorting; IHC, immunohistochemistry; MDSCs, myeloid-derived suppressor cells; PARP, poly(ADP-ribose) polymerase; WT, wild type. 

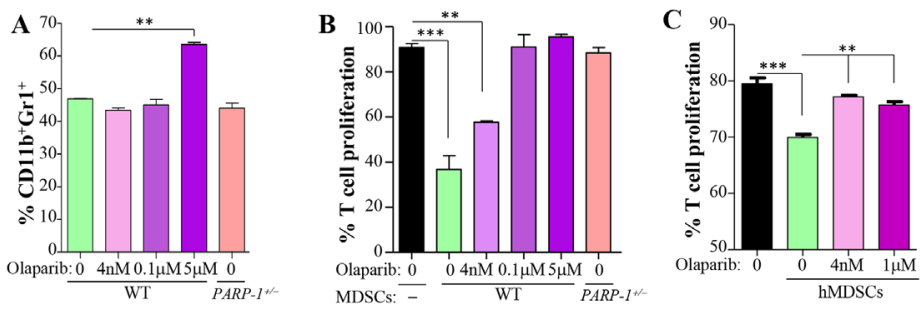

Fig. 5
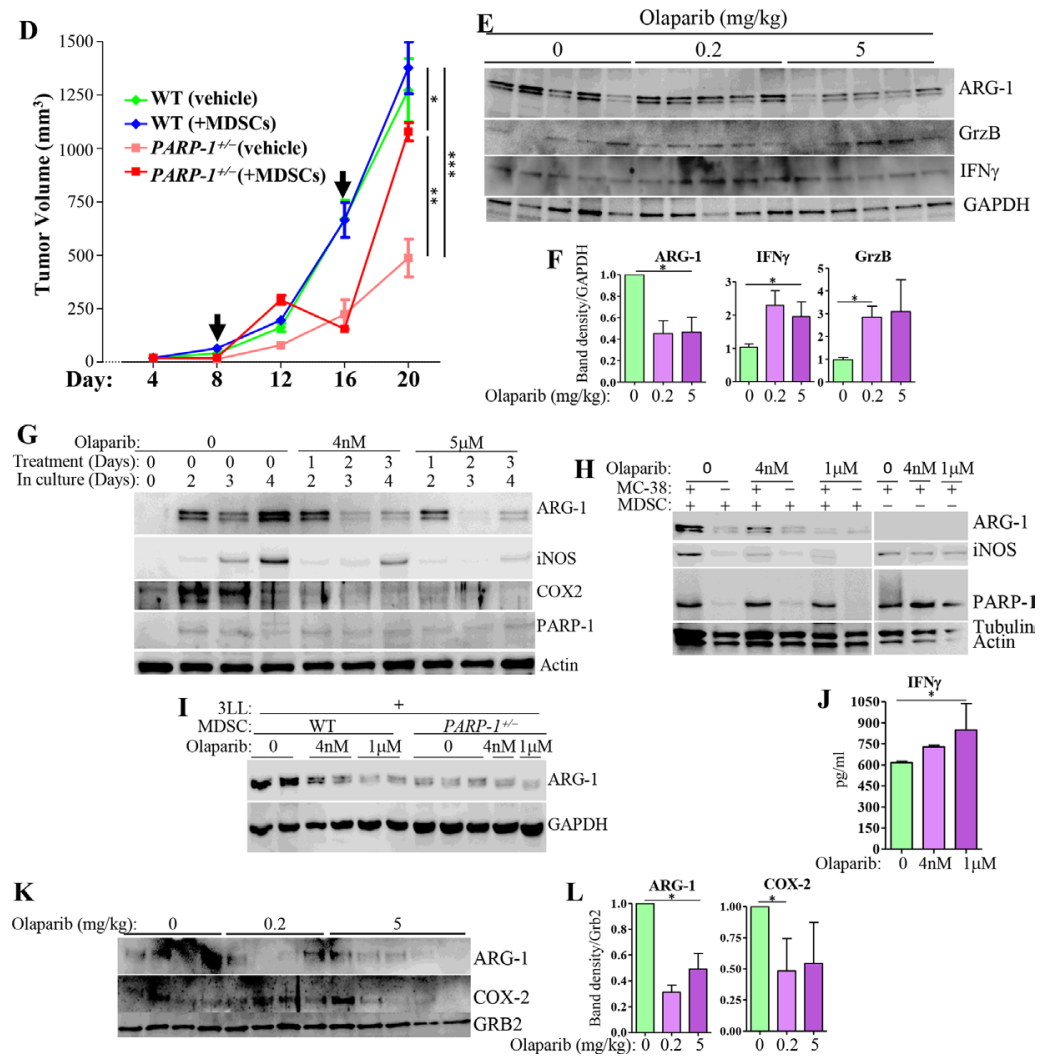

Figure 5 A sub-IC50 concentration of olaparib reduces MDSC suppressive function by blocking the expression of ARG-1/ iNOS/COX-2, and adoptive transfer of WT MDSCs abrogates the protective effects of PARP-1 heterozygosity against tumor burden. BM progenitors from WT or PARP $-1^{+/-}$mice were cultured in differentiation medium; some WT cells were treated with olaparib or vehicle as indicated. After 96 hours, cells were collected and stained with antibodies to CD11b or Gr1 and assessed by FACS (A) or cocultured with CD3/CD28-stimulated and CFSC-labeled WT CD3 ${ }^{+}$T cells for 72 hours followed by an assessment of proliferation by FACS (B). (C) MDSCs derived from human BM were treated, daily, with olaparib for 6 days. Cells were then washed and cocultured with $C D 3^{+} T$ cells for an additional 3 days in the absence of olaparib at an MDSC to T cell ratio of 1:4. T cell proliferation was assessed by FACS. (D) MC-38 cells were engrafted into the left flanks of WT or PARP$1^{+/-}$mice. On days 8 and 16 , mice received, intratumorally, $3 \times 10^{6}$ WT-BM-MDSCs or vehicle. Tumor sizes were measured on the indicated days. (E) $C D 45^{+}$cells isolated from tumors of the different experimental groups ( $n=5$ for each group) were subjected to immunoblot analysis with antibodies to ARG-1, GrzB, IFN $\gamma$, or GAPDH. (F) The intensity of the relevant bands was quantified using ImageJ-Fiji and the average intensity was assessed. (G) BM cells were stimulated and treated with olaparib as indicated. Total protein extracts were subjected to immunoblot analysis with antibodies to ARG-1, iNOS, COX-2, PARP-1, and actin. $(\mathrm{H}) \mathrm{BM}$ cells were treated as stated above and on day $4\left(70 \% \mathrm{Gr} 1^{+}\right)$received $0.5 \mathrm{~mL}$ of fresh medium or medium containing MC-38 cells at a ratio of 2:1 (MC-38/MDSC). The cells received a second treatment with olaparib but with no additional cytokines. Cells were collected after 2 days of treatment and protein extracts were subjected to immunoblot analysis with antibodies to ARG-1, iNOS, PARP-1, tubulin or actin. (I) WT or PARP-1 $1^{+-}$MDSCs were treated as in (G) except that 3LL cells were used instead of MC-38 cells. Protein extracts were subjected to immunoblot analysis with antibodies to iNOS or GAPDH. (J) MDSCs were differentiated in the presence of $4 \mathrm{nM}$ or $1 \mu \mathrm{M}$ olaparib and MC-38 cells. The combinations of cells were cocultured with stimulated T cells in the complete absence of olaparib. Media was then collected and assessed for IFN $\gamma$ by ELISA. All in vitro experiments were conducted at least in triplicates. (K) Tumors were digested and were subjected to Gr1 negative selection followed by CD11b positive selection (to remove tumor cells). Protein extracts were subjected to immunoblot analysis with antibodies to ARG-1, COX-2, or GRB2. The quantification of band intensity is displayed in (L). For A-D, F, J, L: ${ }^{*} \mathrm{p} \leq 0.05,{ }^{* *} \mathrm{p} \leq 0.01,{ }^{* \star *} \mathrm{p} \leq 0.001$. ARG, arginase; BM, bone marrow; CFSE, carboxyfluorescein succinimidyl ester; COX-2, cyclooxygenase-2; FACS, fluorescence-activated cell sorting; GAPDH, glyceraldehyde 3-phosphate dehydrogenase; GRB2, growth factor receptor-bound protein 2; GrzB, Granzyme B; IC50, half maximal inhibitory concentration; IFN $\gamma$, interferon- $\gamma$, iNOS, inducible nitric oxide synthase; MDSCs, myeloid-derived suppressor cells; PARP, poly(ADP-ribose) polymerase; WT, wild type. 
cell function and cytotoxicity markers (eg, interferon- $\gamma$ (IFN $\gamma$ ) and GrzB), further suggesting reduced capacity of immune suppression.

We next conducted a direct examination of the effect of PARP-1 inhibition on the expression of ARG-1 in BM-derived MDSCs as well as other key factors known to play major roles in the function of these cells, such as iNOS and COX-2. ${ }^{11}{ }^{28}$ An expected gradual increase in ARG-1, iNOS, and COX-2 on stimulation with GM-CSF/ G-CSF/IL-6 was observed (figure 5G). MDSCs appeared to be extremely sensitive to treatment with olaparib, as the $4 \mathrm{nM}$ concentration prevented the increase in ARG-1, iNOS, and COX-2 observed in control cells; however, the pattern of the effect on ARG-1 was different from that of iNOS and COX-2. The downregulation of ARG-1 occurred beginning at 2 days of treatment with either concentration of olaparib. Similar results were obtained when MDSC stimulation was mediated by MC-38 (figure $5 \mathrm{H}$ ) or 3LL (figure 5I) cells. The specificity of the ARG-1 and PARP-1 relationship was verified using $P A R P-1^{+-}$MDSCs (figure 5I). Since olaparib can inhibit PARP-2, the addition of the PARPi to PARP-1 $1^{+-}$MDSCs cocultured with cancer cells did not dramatically change the effect on ARG-1 expression, suggesting a lack of involvement of PARP-2 in MDSC function (figure 5I). The effect of PARP inhibition on MDSCs did not only result in an increase in $\mathrm{T}$ cell proliferation but was also accompanied by an increase in IFN $\gamma$ levels in culture medium, suggesting a higher activation of $\mathrm{T}$ cells (figure $5 \mathrm{~J}$ ). Note that, in this experiment, such outcome stemmed from a direct effect on MDSCs as T cells were not exposed to olaparib. The effects of PARP inhibition on the expression of ARG-1 and COX-2 in MDSCs were confirmed in tumor-derived $\mathrm{CD}_{11} \mathrm{~b}^{+} \mathrm{Gr}^{+}$cells by immunoblot analysis (figure $5 \mathrm{~J}, \mathrm{~K}$ ).

\section{The modulatory effect of olaparib on MDSCs function is not strictly dependent on STING but fully independent of DNA damage and PARP-1 trapping}

The mechanism by which PARPi achieves synthetic lethality in BRCA-mutant cancer cells is by trapping PARP-1 on DNA breaks induced by chemotherapy. ${ }^{3}$ Figure 6A shows that PARP-1 remained in the nuclear fraction after treatment with olaparib, suggesting a lack of trapping. Figure $6 \mathrm{~B}$ represents a demonstration of PARP-1 trapping on chromatin following treatment with the DNA-damaging agent VP-16 in Jurkat cells. In the latter cells, the sub-IC50 olaparib concentration promoted little to no PARP-1 trapping onto the chromatin, while the moderate concentration promoted substantial trapping, and these findings reflected the extent of DNA damage as indicated by phosphorylated H2AX ( $\gamma \mathrm{H} 2 \mathrm{AX})$ levels. It is noteworthy that neither concentration alone induced $\gamma \mathrm{H} 2 \mathrm{AX}$, suggesting that the effects of olaparib on MDSC function are independent of DNA damage. Of note, MDSCs were capable of responding to VP-16 as demonstrated by $\gamma \mathrm{H} 2 \mathrm{AX}$ induction (online supplemental figure S5). The absence of a connection between the effects of PARP inhibition on MDSCs and PARP-1 trapping onto the chromatin was confirmed by assessing the levels of PARP-1 and $\gamma \mathrm{H} 2 \mathrm{AX}$ in chromatin fractions of $\mathrm{CD} 11 \mathrm{~b}^{+} \mathrm{Gr}^{+}$ cells isolated from tumors (figure 6C,D).

We next examined whether the STING pathway is associated with the effects of PARP inhibition on MDSC function. We elected to initiate our studies using the BRCA-proficient cell line, HeLa, and the high PARP-1trapping capacity PARPi, talazoparib, which were used to report PARPi-STING activation relationship ${ }^{14}$ by assessing IFN-regulatory factor (IRF)-3 phosphorylation. Figure $6 \mathrm{E}$ shows that IRF-3 phosphorylation was extensive in HeLa cells when treated with $2 \mu \mathrm{M}$ talazoparib, which represents a concentration that is more than 3500 times its IC50 ( 0.57 nM). A similar magnitude of IRF-3 phosphorylation was observed when cells were treated with an equivalent olaparib concentration $(17.5 \mu \mathrm{M})$. Both concentrations exerted maximum inhibition of physiological PARP-1 activity and induced DNA damage as assessed by immunoblot analysis with antibodies to PAR and $\gamma \mathrm{H} 2 \mathrm{AX}$, respectively. These results reproduced the findings reported by Shen et al. ${ }^{14}$ Interestingly, neither the sub-IC50 of olaparib $(4 \mathrm{nM})$ nor that of talazoparib $(0.5 \mathrm{nM})$ induced IRF-3 phosphorylation despite their obvious but partial PARP inhibition. Surprisingly, the sub-IC50 talazoparib concentration caused substantial induction of DNA damage but promoted little to no IRF-3 phosphorylation. The inability of a moderate concentration of olaparib to activate the STING pathway in BRCA-proficient cells is consistent with that reported by Pantelidou et al. ${ }^{13}$ A relatively similar pattern of IRF-3 phosphorylation was observed in MC-38 cells when treated under identical conditions (figure 6F).

Figure 6G shows that STING expression was dispensable for MDSC function as cells derived from BM of TMEM $173^{-/-}$mice exhibited a suppressive capacity similar to that of WT cells. While treatment of TMEM173-- MDSCs with the sub-IC50 concentration of olaparib trended to restore $\mathrm{T}$ cell proliferation, the moderate concentration $(1 \mu \mathrm{M})$ of the drug promoted a recovery that is comparable with that in similarly treated WT MDSCs, suggesting that the effect of PARPi on MDSC function is not strictly dependent on STING. Figure $6 \mathrm{H}$ shows that activation of STING with its agonist, cyclic guanosine monophosphate-adenosine monophosphatec (cGAMP), not only completely blocked the suppressive function of MDSCs, but also enhanced $\mathrm{T}$ cell proliferation higher than that observed in cells stimulated in the absence of MDSCs. The failure of TMEM $173^{-/-}$MDSCs to respond to cGAMP demonstrates the specificity of the relationship between STING and MDSC function. Figure 6I recapitulates the effect of cGAMP on the suppressive function of MDSC derived from human BM, although not to the extent observed in mouse cells. In this particular experiment, only the moderate olaparib concentration promoted $\mathrm{T}$ cell recovery, but more importantly STING activation did not synergize with the PARPi to modulate MDSC function and promote recovery of $\mathrm{T}$ cell proliferation. These results further demonstrate the disconnect between 

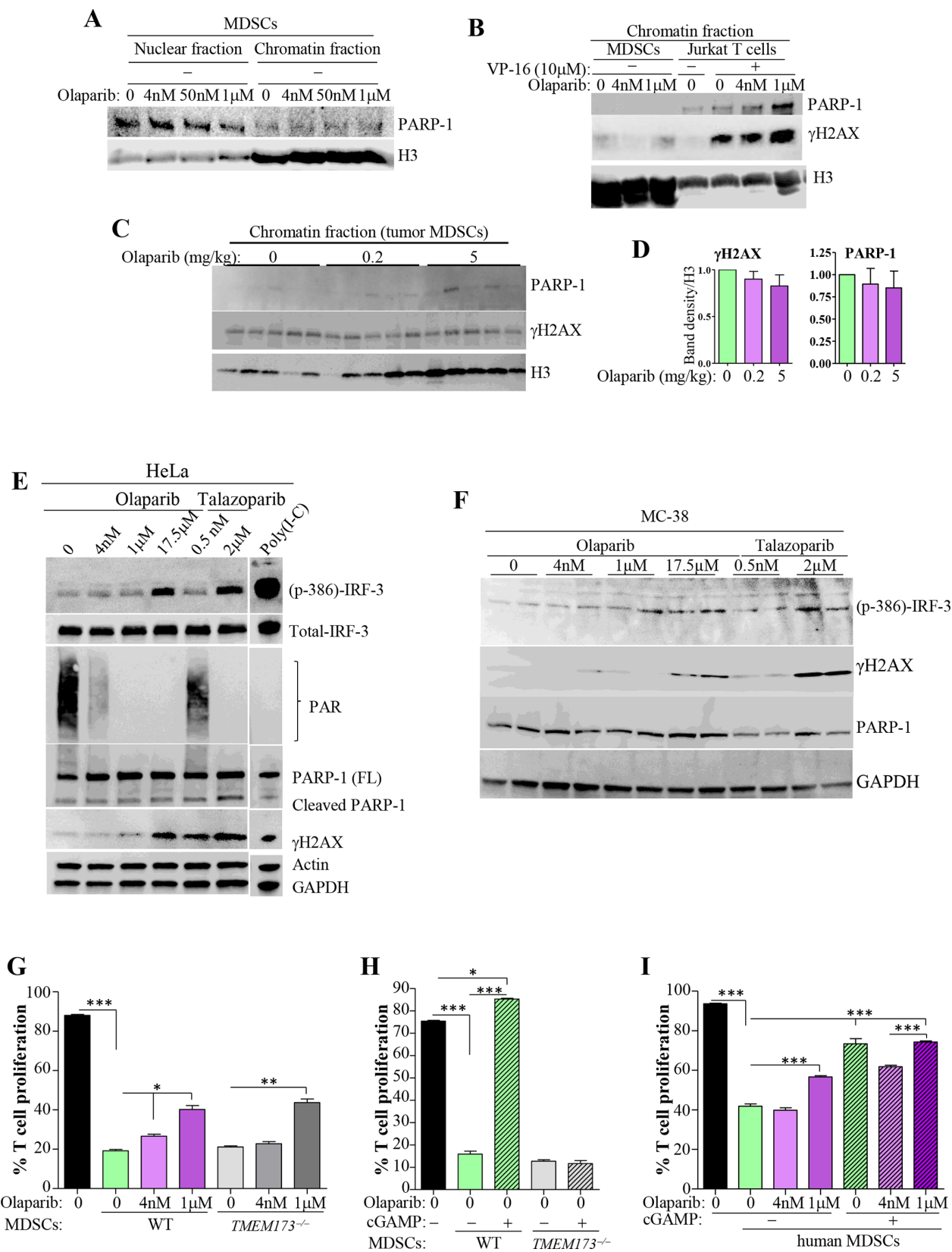

Figure 6 Inhibition of MDSC function by PARPi is partially dependent on STING but independent of DNA damage and trapping of PARP-1 onto the chromatin. (A) WT MDSCs were treated with olaparib for 12 hours. Nuclear and chromatin fractions were subjected to immunoblot analysis with antibodies to PARP-1 or H3. (B) Jurkat T cells were treated with $10 \mu \mathrm{M}$ VP-16 in the presence or absence of olaparib for 1 hour as a positive control for PARP-1 trapping. Chromatin fractions were subjected to immunoblot analysis with antibodies to PARP-1, $\gamma \mathrm{H} 2 \mathrm{AX}$, or $\mathrm{H} 3$. (C) Chromatin fractions from $\mathrm{CD}_{11} \mathrm{~b}^{+} \mathrm{Gr} 1^{+}$cells isolated from tumors of mice that were treated with vehicle or 0.2 or $5 \mathrm{mg} / \mathrm{kg}$ olaparib were subjected to immunoblot analysis with antibodies to PARP-1, $\gamma \mathrm{H} 2 \mathrm{AX}$, or histone H3. (D) The intensity of the relevant bands was quantified using ImageJ-Fiji and the average intensity was assessed. (E) HeLa cells were treated with the indicated concentrations of olaparib or talazoparib for 24 hours. Protein extracts were subjected to immunoblot analysis with antibodies to p-IRF-3, IRF-3, PAR, PARP-1, $\gamma H 2 A X$, actin, or GAPDH. (F) MC-38 cells were treated in a manner similar to that described for HeLa cells. Protein extracts were subjected to immunoblot analysis with antibodies to p-IRF-3, $\gamma \mathrm{H} 2 \mathrm{AX}$, PARP-1, or GAPDH. BM-MDSC derived from WT or TMEM173 ${ }^{-/-}$mice were treated with olaparib $(\mathrm{G})$ or $4 \mu \mathrm{g} / \mathrm{mL}$ cGAMP $(\mathrm{H})$, as described above, prior to coculture with WT T cell at a ratio of 1:4 in the absence of any of the drugs. (I) MDSCs derived from human bone marrow were treated, daily, with olaparib in the presence or absence of an average of $2 \mu \mathrm{g} / \mathrm{mL}$ cGAMP. Cells were then washed and cocultured with CFSE-labeled human CD ${ }^{+} \mathrm{T}$ cells for an additional 3 days in the absence of drugs at an MDSC to T cell ratio of 1:1. T cell proliferation was assessed by FACS. All experiments were conducted at least in triplicates. For $G-1:{ }^{*} p \leq 0.05$, ${ }^{* *} p \leq 0.01$, ${ }^{* \star *} p \leq 0.001$. BM, bone marrow; cGAMP, cyclic guanosine monophosphate-adenosine monophosphate; CFSE, carboxyfluorescein succinimidyl ester; FACS, fluorescenceactivated cell sorting; GAPDH, glyceraldehyde 3-phosphate dehydrogenase; IRF, IFN-regulatory factor; MDSCs, myeloidderived suppressor cells. PAR, poly(ADP-ribose); PARPi, poly(ADP-ribose) polymerase inhibitor; STING, stimulator of interferon genes; WT, wild type. 
PARPi and STING activation in the modulation of MDSC function.

\section{PARPi-based metronomic therapy synergizes with the immune checkpoint inhibitor anti-PD-1 against both MSI ${ }^{\text {high }}$ and MSS tumors with a potential sensitizing effect of anti-PD-1 therapy against MSS tumors}

Although the use of PARPi-based metronomic therapy is unlikely to be adopted in the clinic, it may be ideal for the enhancement of existing immunotherapies, including those targeting PD-1, programmed death-ligand (PD-L) 1 or cytotoxic T-lymphocyte-associated protein (CTLA) $4 .{ }^{29}$ The predicted synergy may increase the proportion of patients that would benefit from immunotherapy especially when considering that only $10 \%-15 \%$ of patients with colon cancer benefit from immunotherapy and those are primarily MSI ${ }^{\text {high }}{ }^{30}$ We first examined the efficacy of a metronomic dose $(0.2 \mathrm{mg} / \mathrm{kg}$ on alternate days) in enhancing the antitumor effect of anti-PD-1 therapy in the MSI ${ }^{\text {high }}$ MC-38 cell-based tumors. As expected, anti-PD-1 and olaparib, individually, significantly reduced the progression of MC-38 tumors in WT mice (figure 7A). Interestingly, the efficacy of the low dose of olaparib in reducing tumor size was comparable with that promoted by anti-PD-1 therapy. Remarkably, however, the combination was substantially more effective at blocking tumor progression. In fact, $78 \%$ of the mice ( 7 out of 9 ) were cured. The efficacy of the metronomic dose of olaparib and anti-PD-1 therapy was reproduced using luciferase-expressing MC-38 cells. Tumors in mice from the different experimental groups were examined using biophotonic imaging (figure 7B), and the luciferase activity signal in the different tumors was determined (figure 7C), further indicating the significant synergy between the metronomic dose of olaparib and anti-PD-1 therapy. Specificity to partial PARP-1 inhibition was verified with the same approach using $P A R P-1^{+/-}$mice (figure 7D). While individual treatments with either antiPD-1 or olaparib partially prevented tumor-associated increase in spleen size, the combination promoted the maintenance of normal-sized spleens (figure 7E). While all forms of treatment reduced MCP-1 and IL-6, with the combination promoting a more pronounced reduction (figure $7 F$ ), the effect on TNF- $\alpha$ was modest. Given the eradication of tumors by the combination of olaparib and anti-PD-1, only a few tumors were large enough to be used for protein extraction followed by immunoblot analysis. While both olaparib and anti-PD-1 immunotherapy induced an increase in PD-L1 in tumors, the combination therapy was associated with a decrease in PD-L1 (figure 7G). The overall activation of caspase 3 and 7 was higher in tumors isolated from animals treated with all forms of treatment. There was a concomitant increase in the DNA damage marker $\gamma \mathrm{H} 2 \mathrm{AX}$ and the cell cycle arrest marker $\mathrm{p} 21 /$ Waf1 in these groups.

It is important to note that the majority of colon cancers are MSS. A good representative model is based on CT-26 cells and is resistant to immunotherapy. ${ }^{31}$ Figure $7 \mathrm{H}$ shows that, indeed, CT-26-based tumors are relatively resistant to anti-PD-1. These tumors also showed a substantial resistance to the metronomic dose of olaparib. However, the combination displayed a remarkable efficacy with potential tumor regression, although not to the level seen in the MSI ${ }^{\text {high }}$ MC-38 cell-based tumor model. Given the resistance of CT-26 cell-based tumors to immunotherapy, these results may also suggest a sensitization of olaparib to anti-PD-1 therapy. Despite the fact that neither treatment affected the increase in spleen size, the combination reduced the size to those of non-tumor-bearing mice (figure 7I). In this model, anti-PD-1 treatment promoted a slight, but statistically significant, increase in the percentage of $\mathrm{CD}_{11} \mathrm{~b}^{+} \mathrm{Gr}-1^{+}$MDSCs, which was reduced to control levels by olaparib treatment (figure $7 \mathrm{~J}$ ). Interestingly, although the tumors were resistant to olaparib, the treatment with the drug increased the percentage of $\mathrm{CD}^{+} \mathrm{T}$ cells in these tumors, which was maintained when combined with anti-PD-1 therapy (figure 7K). Overall, these results suggest a significant synergy between a metronomic dose of olaparib and anti-PD-1 therapy with a potential sensitization given the resistance of the tumor to immunotherapy.

\section{DISCUSSION}

The results of our studies unravel an unexpected role of PARP-1 as an important regulator of MDSC function, providing a paradigm-shifting concept that may be applied to colon cancer and possibly other tumors amenable to immunotherapy. We show that targeting MDSC function with metronomic doses of olaparib provides an impressive antitumor effect alone, but more importantly this approach synergizes with anti-PD-1 immunotherapy and potentially other immune checkpoint inhibitors. The superiority of partial PARP-1 inhibition compared with extensive inhibition was demonstrated in several models, increasing our confidence in the potential relevance to the human disease. The novel concept presented here is that the immunosuppressive MDSCs infiltrating the tumor microenvironment can be effectively modulated using metronomic doses of PARPi, providing an advantage to antitumor immune (eg, $\mathrm{T}$ and natural killer (NK) cells that prevent tumor progression or promote its regression. This was demonstrated by the clear increase in IFN $\gamma$ and GrzB. The addition of immunotherapy, such as anti-PD-1, ensures the maintenance of a robust antitumor response, leading to better outcomes for affected patients. The synergy between PARP inhibition and immunotherapy was efficient in colon tumor models with MSI $^{\text {high }}$ and MSS, although MSI ${ }^{\text {high }}$ tumors responded better. Nevertheless, considering that MSS tumors are considered to be resistant to immunotherapy, our findings suggest that although the PARPi-based metronomic therapy alone is not efficacious, it sensitizes these tumors to immunotherapy. If reproduced in the human condition, our results would allow an increase in the proportion of patients with colon cancer benefitting from 


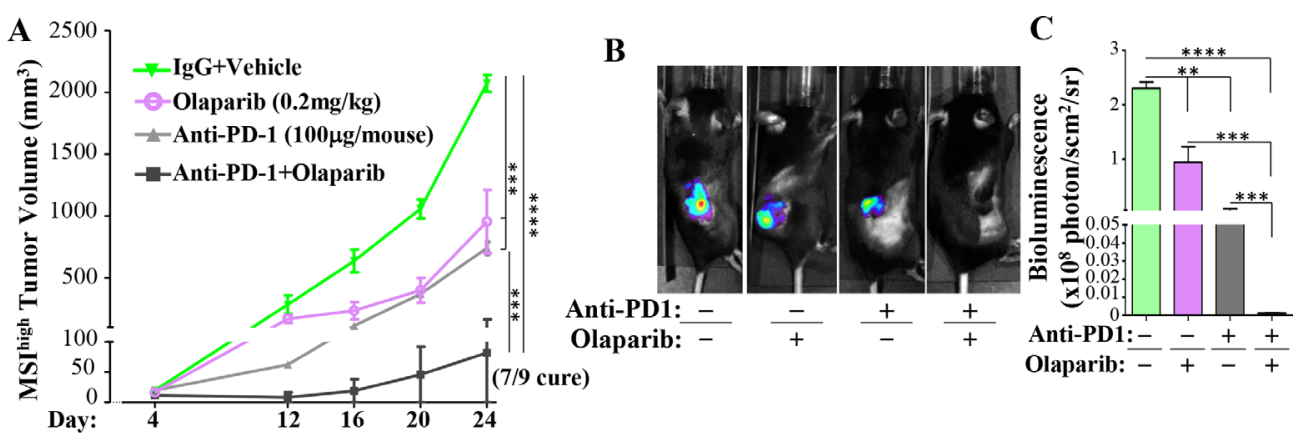

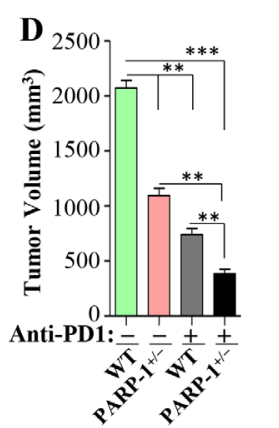

E

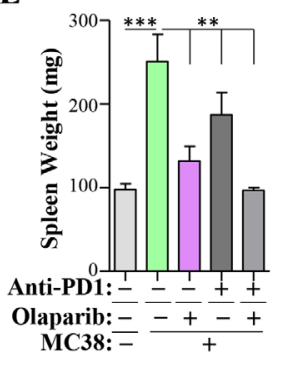

G

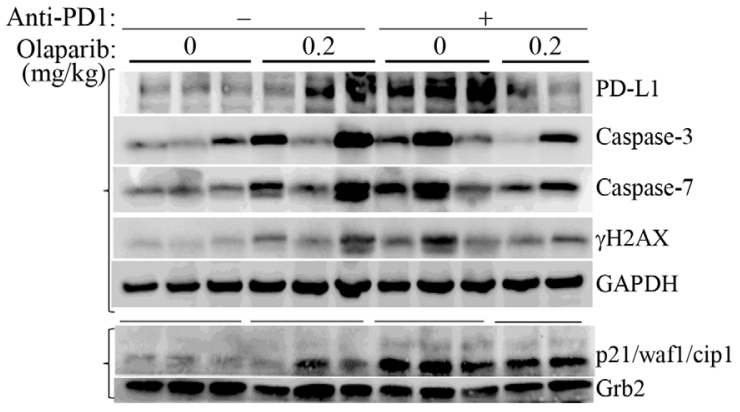

$\mathbf{F}$

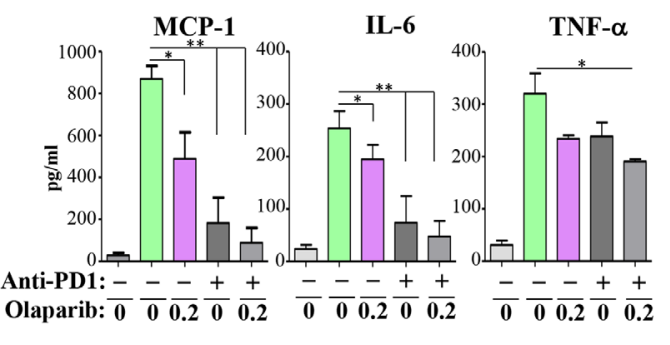

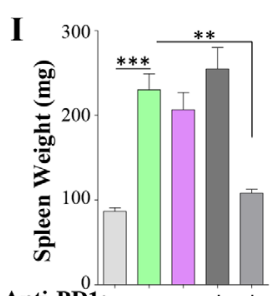

Anti-PD1: - --++

Olaparib: $0 \quad \begin{array}{llllll}0 & 0.2 & 0 & 0.2\end{array}$

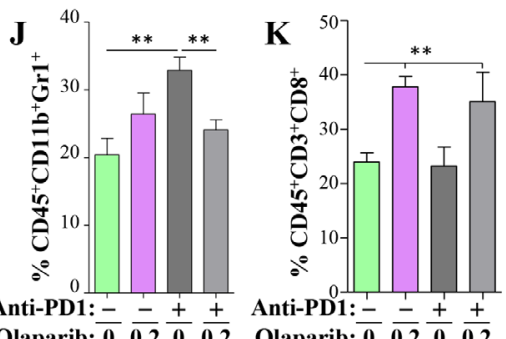

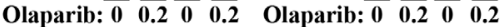

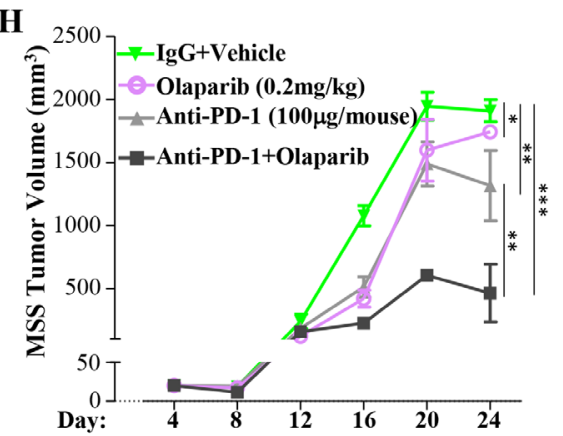

Figure 7 Synergy between a metronomic dose of olaparib and anti-PD-1 therapy to eradicate MC-38 cell-based tumors in mice. (A) MC-38 cells were engrafted into the flanks of WT mice $(n>6)$. When tumors became palpable $\left(\sim 20 \mathrm{~mm}^{3}\right)$, mice were treated with $0.2 \mathrm{mg} / \mathrm{kg}$ olaparib, $100 \mu \mathrm{g}$ anti-PD-1, or a combination of the two agents; mice treated with only olaparib also received an equal amount of IgG isotype. Tumor volumes were then measured. (B,C) GFP-luciferase-expressing MC-38 cells were engrafted into WT mice as in (A). On day 16, tumors were imaged by a whole body IVIS optical imaging system. (B) Representative images of the different experimental groups. (C) Bioluminescence in the region of interest was quantified in photons $/ \mathrm{s} / \mathrm{cm}^{2} / \mathrm{sr}$. (D) MC-38 cells were engrafted into the flanks of WT or PARP- $1^{+/-}$mice. On day 6 , mice were then administered either anti-PD-1 or IgG isotype. Tumor volume was assessed on day 24. (E) Spleen weight of mice from the different groups. (F) Sera from the different mouse groups were assessed for TNF- $\alpha$, IL-6, or MCP-1 by ELISA. (G) Protein extracts from tumors derived from the different experimental groups were subjected to immunoblot analysis with antibodies to PD-L1, cleaved (active) caspase-3 or caspase-7, $\gamma \mathrm{H} 2 \mathrm{AX}$, GAPDH, p21/WAF1, or Grb2. Two left braces represent two different gels using the same samples. $(\mathrm{H}) \mathrm{CT}-26$ cells were engrafted into the flanks of WT mice and then treated with olaparib with antiPD-1 or IgG isotype as described in (A). Tumor volumes were then measured every 4 days. (I) Spleen weight of mice from the different groups. Tumors of the different experimental groups were digested and subjected to staining for MDSCs $(J)$ or CD8 ${ }^{+} T$ cells (K) by FACS. For A, C-F, and H-K: ${ }^{*} \mathrm{p} \leq 0.05,{ }^{* *} \mathrm{p} \leq 0.01,{ }^{* \star *} \mathrm{p} \leq 0.001,{ }^{* * * *} \mathrm{p} \leq 0.0001$. FACS, fluorescence-activated cell sorting; GAPDH, glyceraldehyde 3-phosphate dehydrogenase; GFP, green fluorescent protein; IL-6, interleukin 6; MCP-1, monocyte chemoattractant protein; MDSCs, myeloid-derived suppressor cells; MSI, microsatellite instability; MSS, microsatellite stable; PARP, poly(ADP-ribose) polymerase; PD-1, programmed cell Death protein-1; TNF- $\alpha$, tumor necrosis factor- $\alpha$, WT, wild type. 
existing immunotherapies. Additionally, the concept can be expanded to other immunotherapy-resistant cancers.

MDSC function appears highly sensitive to PARP inhibition and that this effect is not strictly associated with PARP-1 trapping on the chromatin, induction of DNA damage, or STING, suggesting that the mechanism by which PARP inhibition reduces MDSC function is unrelated to its role in DNA repair. The low concentrations of olaparib or other PARPi used in this study do not exert any major effects on the viability of cancer cells, ${ }^{4}$ although non-DNA damage-related physiological PARP activation was affected. PARP inhibition modulates MDSC function by reducing expression of key related factors. An effect on iNOS and COX-2 expression may be predictable because the genes can be regulated by several factors (eg, NF- $\mathrm{KB}$ and STAT6) that others and we have shown to be influenced by PARP-1. ${ }^{18} 32$ However, the mechanism by which PARP-1 inhibition reduces ARG-1 levels appears to be different, as the effects were not evident even after 24 hours of treatment. As stated above, olaparib inhibits both PARP-1 and PARP-2. The addition of the drug to $P A R P-1^{+/-}$MDSCs did not change the effect on ARG-1 expression, suggesting a potentially specific relationship between ARG-1 and PARP-1 but not PARP-2. The requirement for STING in non-tumor cells such as MDSCs, dendritic cells (DCs), or NK cells is not fully elucidated. ${ }^{15} 1633-37$ A recent study provided convincing evidence on the role of STING in MDSC function and demonstrated an intimate relationship with the unfolded protein response pathway. ${ }^{16}$ Our results show that STING expression is dispensable for the suppressive function of BM-derived MDSCs. However, the activation of STING by cGAMP blocked the suppressive function of MDSCs derived from either mouse or human BM. The latter results are in agreement with those of Mohamed $e t$ $a l .{ }^{16}$ We do not believe that our observations and that of Mohamed $e t a l^{16}$ are necessarily contradictory especially when considering that the tumor microenvironment may include yet to be identified factors or pathways in addition to the protein kinase RNA-like ER kinase (PERK) that potentially influence MDSC suppressive function when STING is absent.

The primary goal of clinical trials examining the combination of PARPi and checkpoint blockers is to test whether checkpoint blockers enhance the efficacy of PARPi in a variety of cancers or to explore the potential of high doses of the drugs in increasing mutation rates, which lead to a greater neoantigen generation. The concept proposed herein is different. By targeting primarily MDSCs and potential other immune cells, this concept will not only expand the utility of PARPi approved by the Food and Drug Administration, but will also revive interest in PARPi with a low trapping capacity. Very recently, Jiao et $a l^{88}$ reported that olaparib, at a dose of $50 \mathrm{mg} / \mathrm{kg}$ daily, enhances anti-PD-1 immunotherapy in an allograft breast cancer mouse model, although the PARPi used in the study promoted immunosuppression with a concomitant upregulation of PD-L1 expression. Such effects appeared to be independent of the immune system, since it manifested in a breast cancer xenograft model. In our experimental system, a metronomic dose of PARPi also induced an increase in PD-L1 in tumors, an effect that is not different from that induced by anti-PD-1 immunotherapy. Interestingly, combination therapy was associated with a decrease in PD-L1. Although it is difficult to explain this result, it is tempting to speculate that the lower levels of PD-L1 are associated with the predicted increase in cell killing and proteasomal activity that is known to regulate the fate of the protein. The use of high doses of PARPi may also lead to more genomic instability in cancer cells and, potentially, normal cells. This notion is supported by our results in the $A P C^{M i n /+}$ mouse model, in which PARP-1 gene knockout aggravated tumor burden instead of providing protection as seen with PARP-1 heterozygosity. We reported this dichotomy several years ago in a conference report; ${ }^{39}$ this observation was supported by a recent study reporting that PARP-1 deletion increased AOM/ DSS-induced tumor burden when combined with amplified DNA damage via deletion of the DNA repair gene $\mathrm{O}^{6}$-methylguanine-DNA methyltransferase. ${ }^{24}$ In addition to enhancing the effects of immunotherapy, PARPi may mitigate colitis or cachexia, which are prominent side effects of immunotherapy and cancer in general, respectively. Our observation of the effect of olaparib on cachexia is consistent with a recent study that showed that in a diaphragm and gastrocnemius model of lung cancer with the LP07 adenocarcinoma cell line, PARP-1 (and PARP-2) gene knockout partially protected against cachexia. ${ }^{40}$

In conclusion, we are confident that our results will provide extraordinary opportunities for immediate clinical trials. The results of such trials may ultimately benefit a larger proportion of patients with cancer. Our findings highlight the notion that targeting non-cancer cells such as MDSCs and potentially other immune cells with PARPi may allow the targeting of additional cancer types in addition to BRCA-mutated breast or ovarian cancer.

\section{Author affiliations \\ ${ }^{1}$ The Stanley Scott Cancer Center/Louisiana Cancer Research Center, School of Medicine, Louisiana State University Health Sciences Center, New Orleans, Louisiana, USA \\ 2Department of Microbiology and Immunology, Faculty of Pharmacy, Al-Azhar University, Cairo, Egypt \\ ${ }^{3}$ Department of Pharmacology, Faculty of Pharmacy, Al- Azhar University, Assiut, Egypt \\ ${ }^{4}$ Faculty of Science, Tanta University, Tanta, Egypt}

Correction notice This paper has been updated to revise author affiliations and funding details.

Acknowledgements We would like to acknowledge the technical assistance of Constance Porretta and Dr Arnold Zea (Comprehensive Alcohol Research Center Analytical Core), Dr Luis Marrero (Morphology and Imaging Core), Dr Maria Sanchez-Pino for providing us with the human MDSCs, and Dr Om Prakash (LSUHSC) for the GFP-Luc-expressing plasmid. We would also like to thank Amin Boulares for his special assistance and support. This work is dedicated to the faculty and staff of the Audubon Elementary School (Jefferson Parish Schools, Louisiana) under the leadership of Dr Emily Anderson. 
Contributors MAG, SVI, AFT, and YE conducted most of the experiments and contributed equally to the work. The order of the first authors is based on the significance of the contribution and effort made to generate the data and its analysis and the overall contribution to the writing of the manuscript. MD assisted in the experiments pertinent to MDSCs and immunotherapy and participated in the analysis of the data. AHE assisted AT in some of the animal studies. HL and IB assisted in many of the animal and in vitro studies. DW assisted with tissue processing, FACS, and data analysis. LDV is the pathologist who conducted, in a blinded manner, the IHC and immunofluorescence on tissue sections. AAA assisted in the design and performance of some of the experiments with the syngeneic models, the T cell suppression assays and assisted in the overall analysis of data. $\mathrm{A} 0$ provided part of the support for this work and participated in data discussion. AHB, the principal investigator, designed the study, acquired funding, conducted some of the experiments, analyzed the data and wrote the manuscript.

Funding This work was supported in part by grant RSG- 116608 from the American Cancer Society, and grants P30GM114732; P20CA233374 (overall PD: A0) and P30GM106392 (overall PD: Dr D Kapusta) from the NIH to AHB.

Competing interests None declared.

Patient consent for publication Not required.

Ethics approval Ethical approval was obtained from Louisiana State University Health Sciences Center in May 2017. Experimental protocols were approved by the LSUHSC-IACUC and in accordance with the NIH Guide for the Care and Use of Laboratory Animals.

Provenance and peer review Not commissioned; externally peer reviewed.

Data availability statement Data sharing not applicable as no data sets generated and/or analyzed for this study. All data relevant to the study are included in the article or uploaded as supplementary information. Contact email: hboulr@ Isuhsc.edu.

Supplemental material This content has been supplied by the author(s). It has not been vetted by BMJ Publishing Group Limited (BMJ) and may not have been peer-reviewed. Any opinions or recommendations discussed are solely those of the author(s) and are not endorsed by BMJ. BMJ disclaims all liability and responsibility arising from any reliance placed on the content. Where the content includes any translated material, BMJ does not warrant the accuracy and reliability of the translations (including but not limited to local regulations, clinical guidelines, terminology, drug names and drug dosages), and is not responsible for any error and/or omissions arising from translation and adaptation or otherwise.

Open access This is an open access article distributed in accordance with the Creative Commons Attribution Non Commercial (CC BY-NC 4.0) license, which permits others to distribute, remix, adapt, build upon this work non-commercially, and license their derivative works on different terms, provided the original work is properly cited, appropriate credit is given, any changes made indicated, and the use is non-commercial. See http://creativecommons.org/licenses/by-nc/4.0/.

\section{ORCID iDs}

Mohamed A Ghonim http://orcid.org/0000-0001-9404-2698

Ali H El-Bahrawy http://orcid.org/0000-0001-6116-0428

A Hamid Boulares http://orcid.org/0000-0001-9916-8126

\section{REFERENCES}

1 Veneris JT, Matulonis UA, Liu JF, et al. Choosing wisely: selecting PARP inhibitor combinations to promote anti-tumor immune responses beyond BRCA mutations. Gynecol Oncol 2020;156:488-97.

2 Gourley C, Balmaña J, Ledermann JA, et al. Moving from poly (ADP-ribose) polymerase inhibition to targeting DNA repair and DNA damage response in cancer therapy. J Clin Oncol 2019;37:2257-69.

3 Hopkins TA, Ainsworth WB, Ellis PA, et al. Parp1 trapping by PARP inhibitors drives cytotoxicity in both cancer cells and healthy bone marrow. Mol Cancer Res 2019;17:409-19.

4 Berger NA, Besson VC, Boulares AH, et al. Opportunities for the repurposing of PARP inhibitors for the therapy of non-oncological diseases. Br J Pharmacol 2018;175:192-222.

5 Wang ZQ, Auer B, Stingl L, et al. Mice lacking ADPRT and poly(ADPribosyl)ation develop normally but are susceptible to skin disease. Genes Dev 1995;9:509-20.

6 Claybon A, Karia B, Bruce C, et al. PARP1 suppresses homologous recombination events in mice in vivo. Nucleic Acids Res 2010;38:7538-45.
7 Pommier Y, O'Connor MJ, de Bono J. Laying a trap to kill cancer cells: PARP inhibitors and their mechanisms of action. Sci Trans/ Med 2016;8:362ps 17 .

8 Oumouna-Benachour K, Hans CP, Suzuki Y, et al. Poly(ADPribose) polymerase inhibition reduces atherosclerotic plaque size and promotes factors of plaque stability in apolipoprotein E-deficient mice: effects on macrophage recruitment, nuclear factor-kappaB nuclear translocation, and foam cell death. Circulation 2007;115:2442-50.

9 Ghonim MA, Pyakurel K, Ibba SV, et al. PARP inhibition by olaparib or gene knockout blocks asthma-like manifestation in mice by modulating CD4(+) T cell function. J Trans/ Med 2015;13:225.

10 Ghonim MA, Pyakurel K, Ibba SV, et al. PARP is activated in human asthma and its inhibition by olaparib blocks house dust mite-induced disease in mice. Clin Sci 2015;129:951-62.

11 Gabrilovich DI. Myeloid-Derived suppressor cells. Cancer Immunol Res 2017:5:3-8.

12 Ding L, Kim H-J, Wang Q, et al. Parp inhibition elicits STINGdependent antitumor immunity in BRCA1-deficient ovarian cancer. Cell Rep 2018;25:2972-80.

13 Pantelidou C, Sonzogni O, De Oliveria Taveira M, et al. PARP Inhibitor Efficacy Depends on $\mathrm{CD}^{+} \mathrm{T}$-cell Recruitment via Intratumoral STING Pathway Activation in BRCA-Deficient Models of Triple-Negative Breast Cancer. Cancer Discov 2019;9:722-37.

14 Shen J, Zhao W, Ju Z, et al. PARPi triggers the STING-dependent immune response and enhances the therapeutic efficacy of immune checkpoint blockade independent of BRCAness. Cancer Res 2019;79:311-9.

15 Zhang C-X, Ye S-B, Ni J-J, et al. Sting signaling remodels the tumor microenvironment by antagonizing myeloid-derived suppressor cell expansion. Cell Death Differ 2019;26:2314-28.

16 Mohamed E, Sierra RA, Trillo-Tinoco J, et al. The unfolded protein response mediator PERK governs myeloid cell-driven immunosuppression in tumors through inhibition of sting signaling. Immunity 2020;52:668-82

17 Oumouna-Benachour K, Oumouna M, Zerfaoui M, et al. Intrinsic resistance to apoptosis of colon epithelial cells is a potential determining factor in the susceptibility of the $\mathrm{A} / \mathrm{J}$ mouse strain to dimethylhydrazine-induced colon tumorigenesis. Mol Carcinog 2007;46:993-1002

18 Zerfaoui M, Errami Y, Naura AS, et al. Poly(ADP-ribose) polymerase-1 is a determining factor in Crm1-mediated nuclear export and retention of p65 NF-kappa B upon TLR4 stimulation. J Immunol 2010;185:1894-902.

19 Zerfaoui M, Naura AS, Errami Y, et al. Effects of PARP-1 deficiency on airway inflammatory cell recruitment in response to LPS or TNF: differential effects on CXCR2 ligands and Duffy antigen receptor for chemokines. J Leukoc Biol 2009;86:1385-92.

20 Zerfaoui M, Suzuki Y, Naura AS, et al. Nuclear translocation of p65 NF-kappaB is sufficient for VCAM-1, but not ICAM-1, expression in TNF-stimulated smooth muscle cells: differential requirement for PARP-1 expression and interaction. Cell Signal 2008;20:186-94.

21 Al-Khami AA, Ghonim MA, Del Valle L, et al. Fuelling the mechanisms of asthma: increased fatty acid oxidation in inflammatory immune cells may represent a novel therapeutic target. Clin Exp Allergy 2017;47:1170-84.

22 Naura AS, Datta R, Hans CP, et al. Reciprocal regulation of iNOS and PARP-1 during allergen-induced eosinophilia. Eur Respir $J$ 2009;33:252-62.

23 Boulares AH, Ferran MC, Lucas-Lenard J. Nf-kappaB activation is delayed in mouse L929 cells infected with interferon suppressing, but not inducing, vesicular stomatitis virus strains. Virology 1996;218:71-80.

24 Dörsam B, Seiwert N, Foersch S, et al. PARP-1 protects against colorectal tumor induction, but promotes inflammationdriven colorectal tumor progression. Proc Natl Acad Sci U S A 2018;115:E4061-70.

25 Larmonier CB, Shehab KW, Laubitz D, et al. Transcriptional Reprogramming and Resistance to Colonic Mucosal Injury in Poly(ADP-ribose) Polymerase 1 (PARP1)-deficient Mice. J Biol Chem 2016;291:8918-30.

26 Currey N, Daniel JJ, Mladenova DN, et al. Microsatellite instability in mouse models of colorectal cancer. Can J Gastroenterol Hepatol 2018;2018:1-7

27 Sceneay J, Griessinger CM, Hoffmann SHL, et al. Tracking the fate of adoptively transferred myeloid-derived suppressor cells in the primary breast tumor microenvironment. PLoS One 2018;13:e0196040.

28 Condamine T, Mastio J, Gabrilovich DI. Transcriptional regulation of myeloid-derived suppressor cells. J Leukoc Biol 2015;98:913-22. 
29 Marchetti A, Di Lorito A, Buttitta F. Why anti-PD1/PDL1 therapy is so effective? Another piece in the puzzle. $J$ Thorac Dis 2017;9:4863-6.

30 Arora SP, Mahalingam D. Immunotherapy in colorectal cancer: for the select few or all? J Gastrointest Oncol 2018;9:170-9.

31 Dosset M, Vargas TR, Lagrange A, et al. PD-1/PD-L1 pathway: an adaptive immune resistance mechanism to immunogenic chemotherapy in colorectal cancer. Oncoimmunology 2018;7:e1433981.

32 Hassa PO, Hottiger MO. The functional role of poly(ADP-ribose) polymerase 1 as novel coactivator of NF-kappaB in inflammatory disorders. Cell Mol Life Sci 2002;59:1534-53.

33 Balka KR, Louis C, Saunders TL, et al. Tbk1 and IKKe act redundantly to mediate STING-Induced NF- $\mathrm{KB}$ responses in myeloid cells. Cell Rep 2020;31:107492.

34 Tegtmeyer P-K, Spanier J, Borst K, et al. Sting induces early IFN- $\beta$ in the liver and constrains myeloid cell-mediated dissemination of murine cytomegalovirus. Nat Commun 2019;10:2830.
35 Weiss JM, Guérin MV, Regnier F, et al. The sting agonist DMXAA triggers a cooperation between $T$ lymphocytes and myeloid cells that leads to tumor regression. Oncoimmunology 2017;6:e1346765.

36 Gaidt MM, Ebert TS, Chauhan D, et al. The DNA inflammasome in human myeloid cells is initiated by a STING-Cell death program upstream of NLRP3. Cell 2017;171:1110-24. e18.

37 Liang H, Deng L, Hou Y, et al. Host STING-dependent MDSC mobilization drives extrinsic radiation resistance. Nat Commun 2017;8:8.

38 Jiao S, Xia W, Yamaguchi H, et al. Parp inhibitor upregulates PD-L1 expression and enhances cancer-associated immunosuppression. Clin Cancer Res 2017;23:3711-20.

39 Tarhuni A, Errami Y, El-Bahrawy A, et al. Paradoxical roles of PARP-1 in colon inflammation and tumorigenesis. Faseb $J$ 2015;29:629.11.

40 Chacon-Cabrera A, Mateu-Jimenez M, Langohr K, et al. Role of PARP activity in lung cancer-induced cachexia: effects on muscle oxidative stress, proteolysis, anabolic markers, and phenotype. J Cell Physiol 2017;232:3744-61. 
Correction: Targeting PARP-1 with metronomic therapy modulates MDSC suppressive function and enhances antiPD-1- immunotherapy in colon cancer

Ghonim MA, Ibba SV, Tarhuni AF, et al. Targeting PARP-1 with metronomic therapy modulates MDSC suppressive function and enhances anti-PD-1 immunotherapy in colon cancer. J Immunotherapy Cancer 2021;9:e001643. doi: 10.1136/jitc-2020-001643

This paper has been updated to revise author affiliations and funding details.

Open access This is an open access article distributed in accordance with the Creative Commons Attribution Non Commercial (CC BY-NC 4.0) license, which permits others to distribute, remix, adapt, build upon this work non-commercially, and license their derivative works on different terms, provided the original work is properly cited, appropriate credit is given, any changes made indicated, and the use is non-commercial. See: http://creativecommons.org/licenses/by-nc/4.0/.

(C) Author(s) (or their employer(s)) 2020. Re-use permitted under CC BY-NC. No commercial re-use. See rights and permissions. Published by BMJ.

J Immunother Cancer 2021;9:e001643corr1. doi:10.1136/jitc-2020-001643corr1

D) Check for updates 